\title{
A metabolic inhibitor arms macrophages to kill intracellular fungal pathogens by manipulating zinc homeostasis
}

\author{
Diego C.P. Rossi, ${ }^{1}$ Julio A. Landero Figueroa, ${ }^{2}$ William R. Buesing, ${ }^{1}$ Kathleen Candor, ${ }^{1,2,3}$ Logan T. Blancett, ${ }^{1}$ Heather M. Evans, ${ }^{1}$ \\ Rena Lenchitz, ${ }^{1}$ Bradford L. Crowther III, ${ }^{1,4}$ Waleed Elsegeiny, ${ }^{5}$ Peter R. Williamson, ${ }^{5}$ Jan Rupp, ${ }^{6}$ and Ceorge S. Deepe Jr. ${ }^{1}$
}

'Division of Infectious Diseases, College of Medicine and 2University of Cincinnati/Agilent Technologies Metallomics Center of the Americas, Department of Chemistry, University of Cincinnati, Cincinnati, Ohio, USA. ${ }^{3}$ Immunology Graduate Program and ${ }^{4}$ Department of Molecular Genetics, Biochemistry, and Microbiology, University of Cincinnati College of Medicine, Cincinnati, Ohio, USA. ${ }^{5}$ Laboratory of Clinical Immunology and Microbiology (LCIM), National Institute of Allergy and Infectious Diseases (NIAID), NIH, Bethesda, Maryland, USA. ${ }^{6}$ Department of Infectious Diseases and Microbiology, German Center for Infection Research, Hamburg-Lübeck-Borstel-Riems, Lübeck, Germany.

\begin{abstract}
Macrophages deploy numerous strategies to combat invasion by microbes. One tactic is to restrict acquisition of diverse nutrients, including trace metals, a process termed nutritional immunity. Intracellular pathogens adapt to a resource-poor environment by marshaling mechanisms to harvest nutrients. Carbon acquisition is crucial for pathogen survival; compounds that reduce availability are a potential strategy to control intracellular replication. Treatment of macrophages with the glucose analog 2-deoxy-D-glucose (2-DC) armed phagocytes to eliminate the intracellular fungal pathogen Histoplasma capsulatum in vitro and in vivo. Killing did not rely on altering access to carbon-containing molecules or changes in ATP, ER stress, or autophagy. Unexpectedly, 2-DG undermined import of exogenous zinc into macrophages, decreasing the quantity of cytosolic and phagosomal zinc. The fungus perished as a result of zinc starvation. This change in metal ingress was not ascribed to a defect in a single importer; rather, there was a collective impairment in transporter activity. This effect promoted the antifungal machinery of macrophages and expanded the complexity of 2-DC activities far beyond manipulating glycolysis. Mechanistic metabolic studies employing 2-DG will have to consider its effect on zinc transport. Our preclinical data support consideration of this agent as a possible adjunctive therapy for histoplasmosis.
\end{abstract}

\section{Introduction}

Macrophages are a key constituent of innate immunity; these cells are specialized to kill or orchestrate the elimination of microbial invaders. Depending on the stimulus, macrophages can promptly reprogram their glucose metabolism in response to a threat. Glucose metabolism is decisive in building an effective defense response, not only to supply energy but also to directly activate antimicrobial responses (1). Despite the large antimicrobial defense repertoire of these phagocytes, intracellular pathogens have evolved to live and replicate in this unwelcoming environment (2). Among mammalian professional phagocytes, only macrophages offer a replicative niche for yeast cells of the pathogenic fungus Histoplasma capsulatum. In this subset of phagocytes, $H$. capsulatum survives antimicrobial defenses and replicates as an intracellular pathogen. This thermally dimorphic fungus causes histoplasmosis, a disease of immunocompromised and immunocompetent individuals, which is the most common endemic pulmonary mycosis in the United States (3). Once inside the macrophage, the compartment where H. capsulatum is housed is limited in nutrients. This

Conflict of interest: The authors have declared that no conflict of interest exists. Copyright: () 2021, American Society for Clinical Investigation.

Submitted: December 28, 2020; Accepted: July 6, 2021; Published: August 16, 2021.

Reference information: / Clin Invest. 2021;131(16):e147268.

https://doi.org/10.1172/JCl147268. new environment poses a great challenge for the fungus and forces it to adapt in order to grow (4-7).

Although the glucose metabolic change in macrophages is designed to protect the host from infections, some intracellular pathogens hijack carbon sources for their own benefit. Brucella abortus and Mycobacterium tuberculosis promote intracellular survival and replication by using lactate derived from host glycolysis as a carbon source $(8,9)$. The sophisticated secretion system that Legionella pneumophila possesses undermines the host's mitochondrial respiration and increases glycolysis, which sustains bacterial growth (10). H. capsulatum relies on glycolytic substrates from the host to fuel gluconeogenesis, which is crucial for the fungus to prevail within macrophages (7). Consequently, the use of chemical compounds that reduce the availability of carbon sources or inhibit host glycolysis is a potential strategy for macrophages to cope with $H$. capsulatum infection.

Aside from the generation of reactive oxygen or nitrogen species, the host employs other strategies to prevent intracellular growth of microbes. One of these alternatives is nutritional immunity, in which the host cell deprives the microbe of an essential nutrient. This mechanism may be triggered by immune mediators that prevent microbial access to necessary elements (11). IFN- $\gamma$ activation of phagocytes causes restriction of phagosomal copper and iron, starving H. capsulatum of these elements $(6,12)$. In a similar vein, GM-CSF limits zinc import into phagosomes, thus depriving the organism of this metal and hampering proliferation (13). 
Another approach is to manipulate metabolism to change the behavior of the host cells. Glucose analogues have been extensively used to inhibit glucose metabolism, especially in cancer cells. Of the many glucose analogues, 2-deoxy-D-glucose (2-DG) is effective in the inhibition of glucose metabolism and ATP production (14). This compound has been frequently employed to examine the influence of glycolysis on cellular function (14). Because of its unique structure, 2-DG interferes with N-linked glycosylation of proteins, causing stress in the ER $(14,15)$ leading to autophagy (16).

In vitro, $H$. capsulatum infection triggers a robust glycolytic response in macrophages (17). Moreover, this fungus causes a positive signal by $\left[{ }^{18} \mathrm{~F}\right]$-fluorodeoxyglucose PET in patients with histoplasmosis, thus suggesting that glycolysis persists in infected tissues $(18,19)$. Therefore, we hypothesized that inhibition of glycolysis by 2-DG in infected macrophages would influence the survival of $H$. capsulatum.

Indeed, 2-DG manipulated the macrophage response such that it promoted killing of $H$. capsulatum. Unexpectedly, the killing mechanism of 2-DG was unrelated to a decrease in glycolysis, ATP production, ER stress, or autophagy. 2-DG-mediated elimination of $H$. capsulatum by macrophages was a consequence of zinc starvation. 2-DG selectively impaired import of this trace metal into the macrophages and subsequently the yeast. This glucose analog inhibited the capacity of macrophages to shuttle zinc intracellularly despite upregulation in zinc importers. Thus, a compound that is frequently employed to study glycolysis exerted an action on macrophages that has yet to be described, to our knowledge. This finding may explain how 2-DG acts to limit intracellular growth of other intracellular pathogens, but also compounds the complexity of its mechanism of action.

\section{Results}

2-DG arms macrophages to kill $H$. capsulatum in vitro and in vivo. Using bone marrow-derived macrophages (BMDMs), 2-DG concentrations ranging from 0.5 to $5 \mathrm{mM}$ inhibited the growth of the fungus at an MOI of 1 and 5 at 24 hours and 48 hours as assessed by CFUs or XTT (2,3-bis-[2-methoxy-4-nitro-5sulfophenyl]-2H-tetrazolium-5-carboxanilide) assay. These 2 assays produced comparable results (Figure 1A). When tested in infected human alveolar and peripheral blood-derived macrophages, the results were similar (Figure 1B). To determine whether 2-DG exerted fungicidal or fungistatic activity, we cultured BMDMs for 2, 24, and 48 hours after infection. At 24 and 48 hours, CFUs incubated with 2-DG were less than that found after 2 hours (Figure 1C); therefore, 2-DG exerted fungicidal activity. Despite this potent activity, the effect of 2-DG waned after 24 hours. CFUs from 2-DG-exposed cells increased 2- to 3-fold, similar to controls (Figure 1A: MOI 5 and Figure 1C). 2-DG analogs, 2-fluoro-2-deoxy-D-glucose (2-FDG) and 2-deoxy-2-fluoro-D-mannose (2-FDM), also inhibited growth of $H$. capsulatum by macrophages (Figure 1D). We analyzed the time course of yeast cell growth inhibition. The viability of yeast cells diminished modestly as early as 3 hours after exposure to 2-DG and continued a progressive decline thereafter (Figure 1E). BMDMs differentiated with GM-CSF or M-CSF are phenotypically distinct. GM-CSF promotes a proinflammatory phenotype, whereas exposure to M-CSF causes differentiation to a reparative phenotype (20). GM-CSF- or M-CSF-differentiated macrophages exposed to 2-DG killed $H$. capsulatum equivalently (Figure 1F).

To verify that 2-DG did not kill yeast cells directly, we exposed fungal elements to 2-DG for 24 hours and assessed survival. $H$. capsulatum exhibited more than $90 \%$ viability in the absence or presence of 2-DG (Figure 1G). Another possible explanation for the effect of 2-DG is that it damages yeast cells such that they became susceptible to the innate killing mechanisms of BMDMs. To test this, yeast cells were pretreated with 2-DG or medium for 6 hours. After successive washes, yeasts were fed to BMDMs. Yeast cells preexposed to 2-DG survived as well as those incubated in medium (Figure 1H). 2-DG did not reduce viability of BMDM (Figure 1I) or interfere with phagocytosis (Figure 1J). To support the latter, supernatants from infected macrophages were collected and the number of yeasts quantified after 2 hours of infection. Equal numbers of yeasts were present in supernatants of 2-DG-treated and untreated BMDMs (Supplemental Figure 1A; supplemental material available online with this article; https://doi.org/10.1172/JCI147268DS1).

The influence of 2-DG on the intracellular milieu of macrophages was analyzed to determine whether killing was accompanied by changes in cytocidal mediators. First, we inquired whether ROS contributed to the 2-DG killing mechanism. Infected BMDMs were incubated with 2-DG in the presence of apocynin (APO), a specific inhibitor of mammalian NADPH oxidase. This ROS inhibitor did not abolish fungal death in 2-DG-exposed macrophages (Figure 2A), although in parallel experiments the same concentration reduced ROS to zymosan (Supplemental Figure 2A). We asked whether 2-DG altered the nature of the phagosome by changing its acidity or alkalinity. $H$. capsulatum tightly regulates the phagosomal $\mathrm{pH}$ in order to survive $(21,22)$; the phagosomal $\mathrm{pH}$ was equivalent between controls and 2-DGtreated cells (Figure 2B).

Since 2-DG is phosphorylated by macrophage hexokinase (HK) to form 2-DG-6-phosphate (2-DG6P), another possible explanation is that 2-DG6P is driving the death of H. capsulatum. This has been observed in L. pneumophila (23). 2-DG6P enters the bacteria via a hexose-phosphate transporter; once inside, 2-DG6P interferes with growth. We asked whether a similar mechanism was operative for $H$. capsulatum yeasts. After 24 hours, the survival of yeasts in BMDMs incubated with 2-DG6P was $90 \%$ (Figure 2C). Another consideration is that the intracellular environment could stimulate $H$. capsulatum yeast to express a hexose-phosphate transporter and facilitate 2-DG6P entry. Consequently, we infected BMDMs with $H$. capsulatum yeasts for 6 hours, lysed the BMDMs, and incubated the recovered cells with 2-DG6P. Under these conditions, 2-DG6P did not alter $H$. capsulatum survival (Figure 2C).

Based on in vitro observations, we investigated the effect of 2-DG in vivo. Animals that were intranasally (i.n.) infected and treated with daily doses of 2-DG exhibited a significant reduction in fungal burden at 3 and 7 days of infection (Figure $3 \mathrm{~A}$ ). This effect was independent of sex: treatment reduced fungal burden in male and female mice. To determine whether the effect of 2-DG depended on the presence of $\mathrm{T}$ cells or neutrophils, we depleted 
A

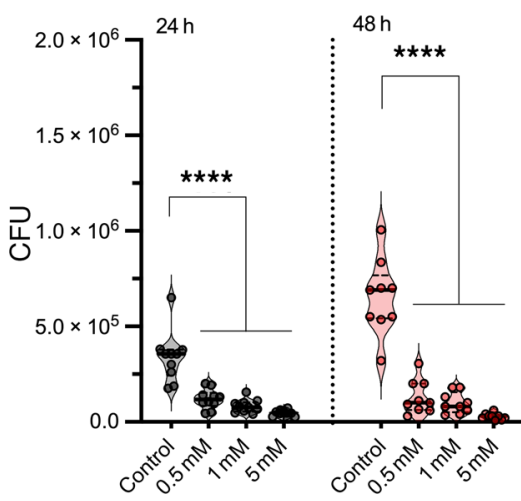

MOI 5

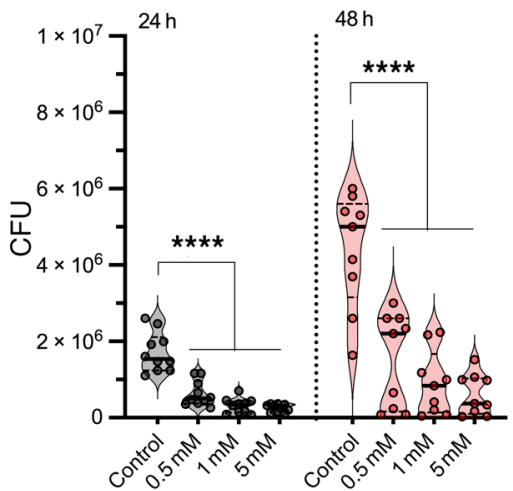

XTT: MOI 5

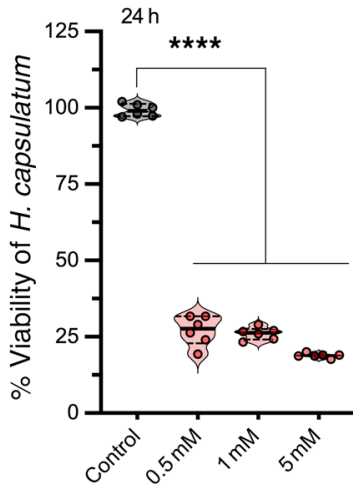

B

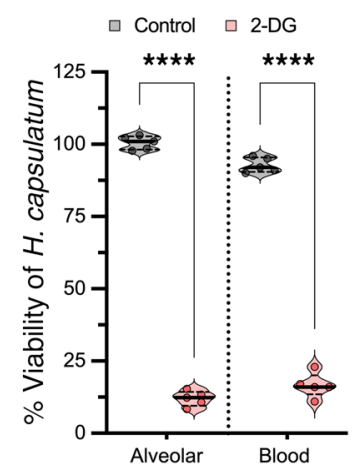

C

D

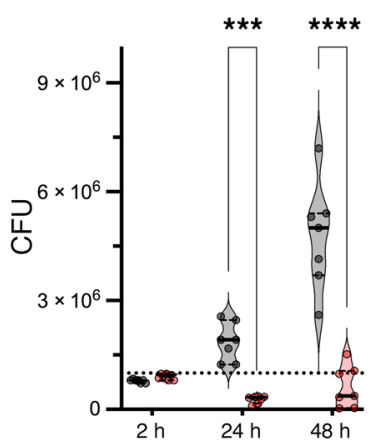

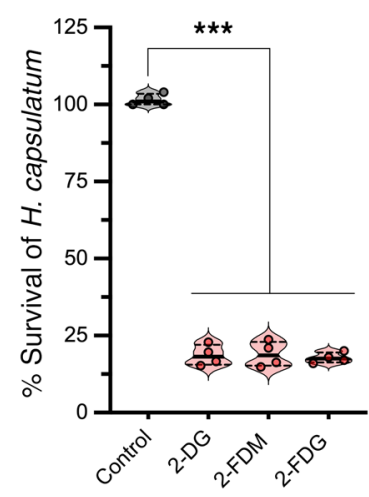

E
$\mathbf{F}$

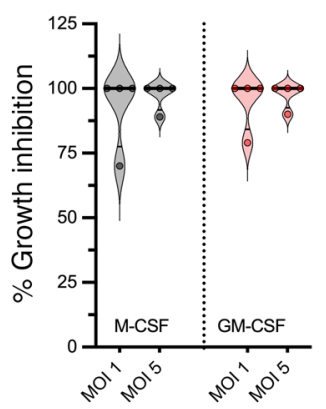

G

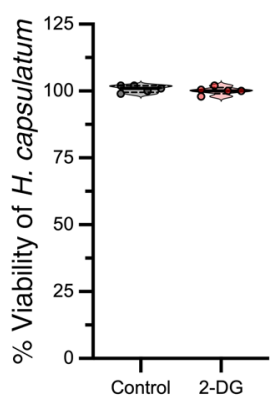

H

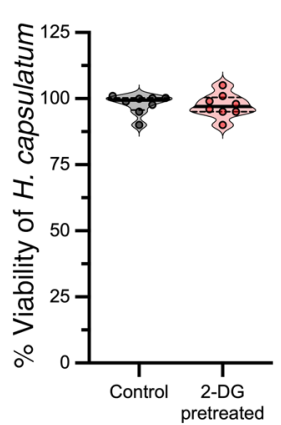

I

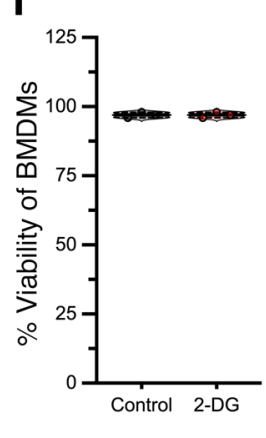

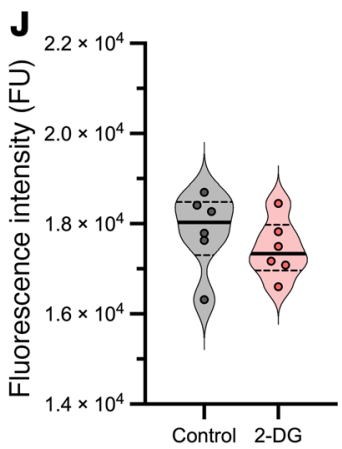

Figure 1. Macrophages fortified by 2-DG kill intracellular H. capsulatum. (A) CFU and XTT assay from BMDMs infected and treated with 2-DG. One-way ANOVA with Tukey's multiple-comparison test. (B) XTT assay from infected human macrophages, MOI 1:5, treated with 5 mM 2-DG. Assay was done after 24 hours of infection. Two-tailed $t$ test. (C) CFU assay from infected BMDMs, MOI 1:5, treated with $5 \mathrm{mM}$ 2-DG. Line represents the initial inoculum, $1 \times$ 106. Two-way ANOVA with Sidak's multiple-comparison test. (D) XTT assay from BMDMs infected (MOI 1:5) and treated with 2-DG (5 mM), 2-FDM (5 mM), and 2-FDG (5 mM) for 24 hours. One-way ANOVA with Tukey's multiple-comparison test. (E) XTT kinetics of $H$. capsulatum viability from BMDMs treated with $5 \mathrm{mM}$ 2-DG. (F) CFU assay from infected BMDMs treated with $5 \mathrm{mM}$ 2-DG. Two-tailed $t$ test. (G) XTT assay from yeasts treated with $5 \mathrm{mM}$ 2-DG for 24 hours. Two-tailed $t$ test. (H) Before infection, yeasts were preincubated with $5 \mathrm{mM}$ 2-DG for 6 hours. After several washes, BMDMs were infected, MOI 1:5. After 24 hours, XTT assays were performed. Two-tailed $t$ test. (I) XTT assay from BMDMs treated with 5 mM 2-DC. Two-tailed $t$ test. (J) Phagocytosis assay from infected BMDMs treated with 5 mM 2-DG. Two-tailed $t$ test. Data are representative of 10 (A), 6 (A: XTT, and J), 5 (B, E, G, and I), 7 (C), 4 (D and F), or 8 (H) independent experiments. Violin plots show the median (line) and quartiles (dashed line). ${ }^{* *} P<0.001 ;{ }^{* * *} P<0.0001$.

mice of these respective cell populations and infected them with H. capsulatum. 2-DG reduced the fungal burden in mice lacking T cells or neutrophils comparable to that of mice with these populations (Figure 3, B and C). Thus, 2-DG did not require $\mathrm{CD} 4^{+}$or $\mathrm{CD} 8^{+}$ $\mathrm{T}$ cells or neutrophils to exert its fungicidal activity. We asked whether 2-DG would have a similar effect on established infection. Infected mice were treated on day 3 of infection and contin- ued through day 6. Recipients of 2-DG exhibited a sharp decrease in fungal burden (Figure 3D).

Glycolysis inhibition and ATP depletion are not required for the effect of 2-DG. Blocking of glycolysis by 2-DG occurs when this chemical is phosphorylated by HK and forms 2-DG6P, which is not metabolized further; this inhibition results in ATP depletion (Figure 4A). We examined whether the same concentration of 

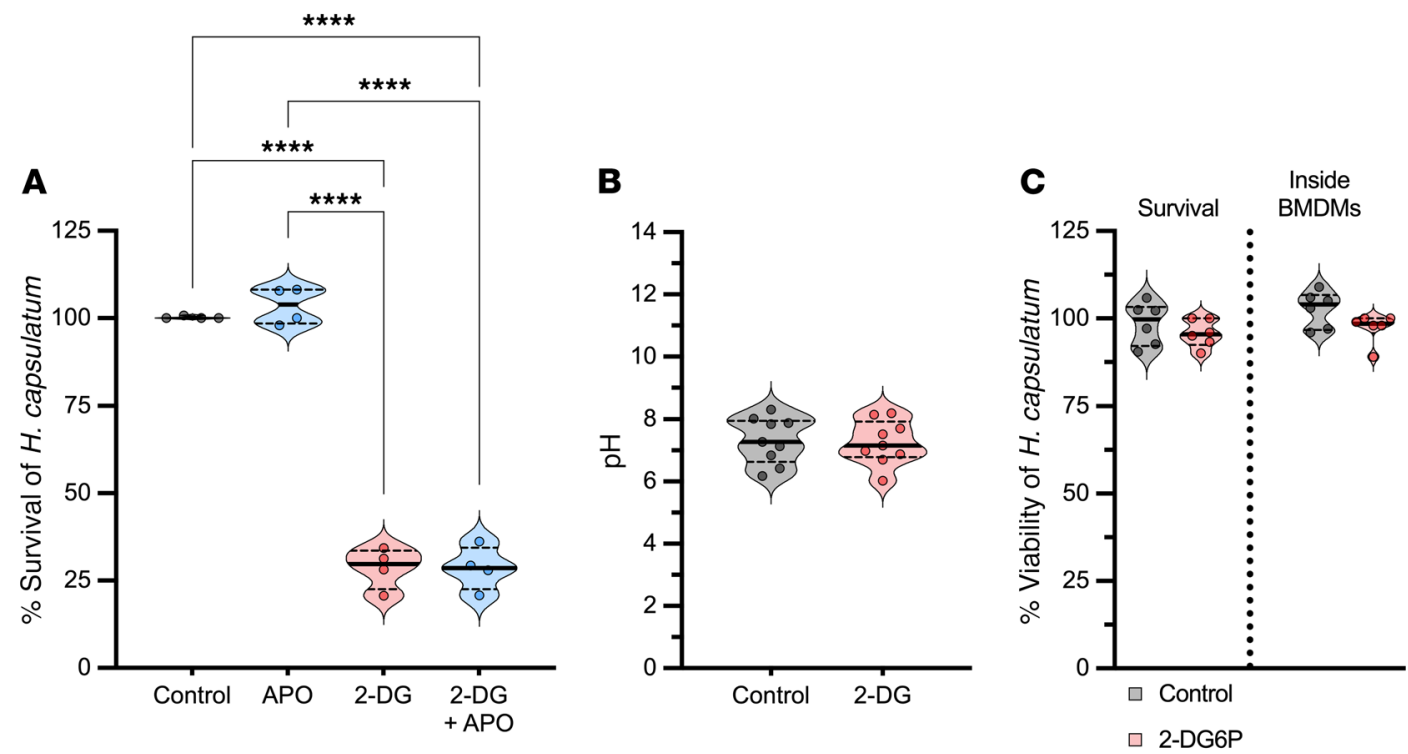

Figure 2. The impact of 2-DG on the intracellular milieu of BMDMs. (A) XTT assay from infected BMDMs treated with $5 \mathrm{mM} 2-\mathrm{DC}$ or 200 $\mu \mathrm{M}$ apocynin 24 hours after infection. One-way ANOVA with Tukey's multiple-comparison test. (B) Intracellular pH measurement of infected BMDMs after 6 hours of 2-DC treatment (5 mM). Two-tailed $t$ test. (C) XTT (survival) assay from infected BMDMs, MOI 1:5, treated with 5 mM 2-DG6P. Inside of BMDMs, after 6 hours of infection, yeasts were recovered from BMDMs and treated with $5 \mathrm{mM}$ 2-DG6P for 24 hours; subsequently, XTT assays were performed. Two-way ANOVA with Sidak's multiple-comparison test. Data are representative of 4 (A), 5 (B), or 6 (C) independent experiments. Violin plots show the median (line) and quartiles (dashed line). ${ }^{* * *} P<0.0001$.

2-DG used to kill H. capsulatum impaired glycolysis. After 3 hours of incubation with 2-DG, infected BMDMs manifested less glycolysis and mitochondrial respiration (Figure 4, B and C).

Accumulation of 2-DG6P causes allosteric and competitive inhibition of HK (24). Measurement of HK activity of infected BMDMs treated with 2-DG revealed a modest yet significant $(P$ $<0.005)$ reduction in HK activity, similar to 3-bromopyruvate (3-BP), a pyruvate analogue that inhibits HK activity (Figure 4D). BMDMs treated with 3-BP did not display fungal killing (Figure $4 \mathrm{~F})$. Thus, HK inhibition was not associated with the antifungal activity of 2-DG.

We ascertained whether restoration of ATP production altered the activity of 2-DG. Dichloroacetate (DCA) blocks mitochondrial pyruvate dehydrogenase kinase (PDK), which inhibits pyruvate dehydrogenase $(\mathrm{PDH})$. This enzyme is crucial for conversion of pyruvate to acetyl-CoA, and thereby increases the influx of pyruvate from glycolysis into the TCA cycle (25). Although DCA reestablished ATP generation (Figure 4F), the 2-DG killing effect was unchanged (Figure 4G).

We determined whether glucose is a necessary constituent for the killing activity mediated by 2-DG. Cells were cultured in glucose- or galactose-containing medium. The latter hexose is a much weaker inducer of glycolysis than glucose (26). The effect of 2-DG depended on the presence of glucose but not galactose in the medium (Figure $4 \mathrm{H}$ ). The less glucose in the medium the more inefficient the effect of 2-DG (Figure 4I).

Autophagy and ER stress are not required for $H$. capsulatum killing. Macroautophagy, hereafter referred to as autophagy, is a recycling and lysosomal degradative process. This process reinforces cellular homeostasis by removing defective organelles and protein aggregates. Autophagy is involved in host immune defenses and is responsible for eliminating intracellular patho- gens in a process termed xenophagy (27). Autophagosomes are a key structure in autophagy, consisting of a spherical structure with double-layer membranes that are decorated with a protein named microtubule-associated protein 1A/1B-light chain 3 (LC3). During autophagosome maturation, a cytosolic form of LC3 (LC3-I) is conjugated to phosphatidylethanolamine (LC3II), and this lipidated form of LC3 is recruited to autophagosomal membranes. A noncanonical form of autophagy named LC3-associated phagocytosis (LAP) differs in several ways from classical autophagy, although it shares various components of the autophagic machinery. In LAP, the autophagosome contains only one membrane and is involved with LC3-II. H. capsulatum prompts the recruitment of LC3-II to a single-walled membrane in mouse and human cells $(17,28)$. Autophagy induced by $2-D G$ is a mechanism that reportedly arms macrophages to kill L. pneumophila (29). We investigated whether 2-DG acted in a similar fashion. First, we searched for the presence of LC3-II in infected BMDMs treated with 2-DG or vehicle. Both sets of infected BMDMs expressed LC3-II regardless of whether they did or did not receive 2-DG (Figure 5A). Second, we explored whether 2-DG changed the nature of the phagosomal membrane from a single membrane to a double membrane by using transmission electron microscopy. Infected BMDMs treated with 2-DG contained yeast cells enclosed by only one membrane, suggesting that 2-DG did not modify the yeast cell compartment (Figure $5 \mathrm{~B}$ ).

To affirm that modulation of LAP by 2-DG is not involved in the killing, we utilized mice lacking autophagy-related gene 5 (ATG5). This member of the autophagy gene family is considered a fundamental protein that is required for both autophagy and LAP (30). We infected ATG5-deficient or -sufficient BMDMs and treated them with 2-DG. Fungal killing exerted by 2-DG was unaffected by the absence of ATG5 in BMDMs. Thus, 
A

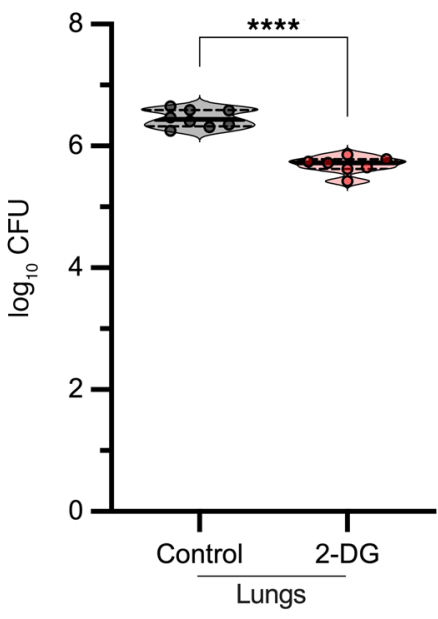

7 Days

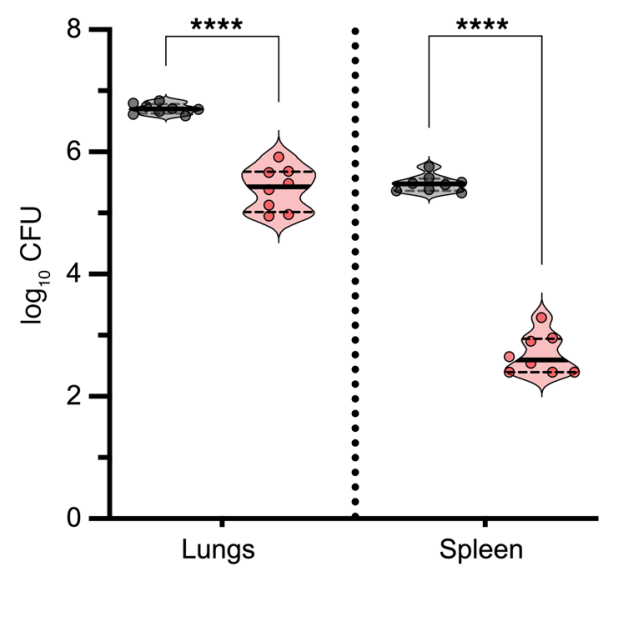

$\square$ Control

口 2-DG
B

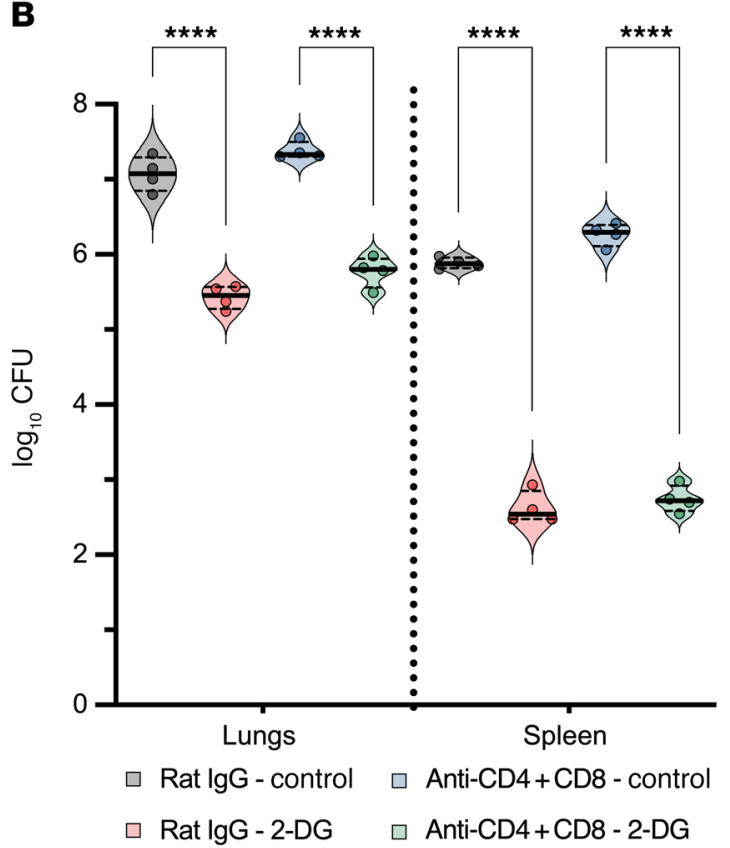

C

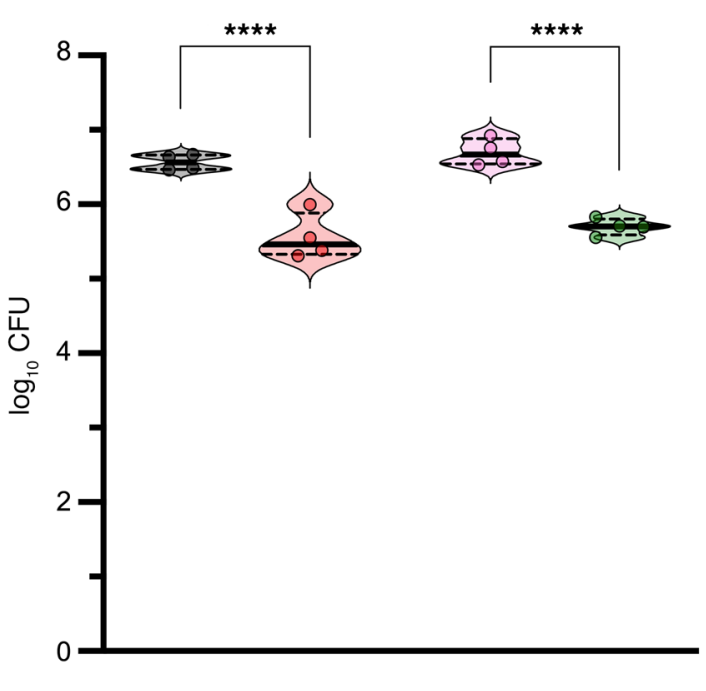

D

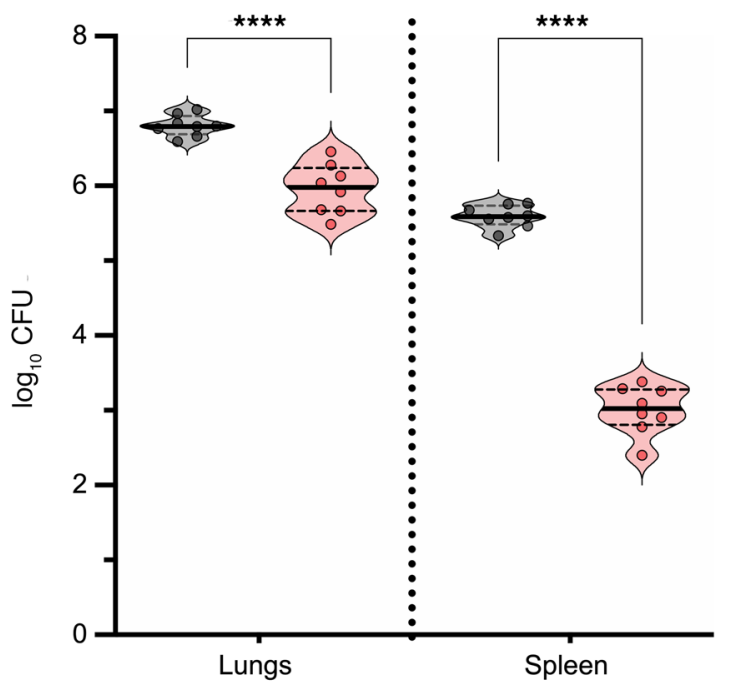

$\square$ Control

口 2-DG
Figure 3. Activity of 2-DG in vivo. (A) CFU of lungs and spleens of mice treated with 2-DG for 3 and 7 days. Two-tailed $t$ test. (B) CFU of lungs and spleen of CD4and CD8-depleted mice treated with 2-DG for 7 days. One-way ANOVA with Tukey's multiple-comparison test. (C) CFU of lungs from neutrophil-depleted mice treated with 2-DG. One-way ANOVA with Tukey's multiplecomparison test. (D) CFU of lungs and spleens from infected controls and 2-DG-treated mice. Mice received the first dose of 2-DG on day 3 of infection, and treatment was continued until day 6 . On day 7 after infection, lungs and spleen were harvested. Two-tailed $t$ test. Data are representative of 7 (A and $\mathbf{D}$ ) or 4 ( $\mathbf{B}$ and $\mathbf{C}$ ) independent experiments. Violin plots show the median (line) and quartiles (dashed line). ${ }^{* * *} P<0.0001$. 
A

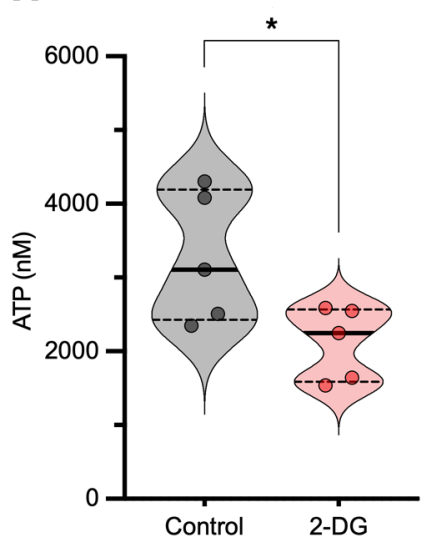

D

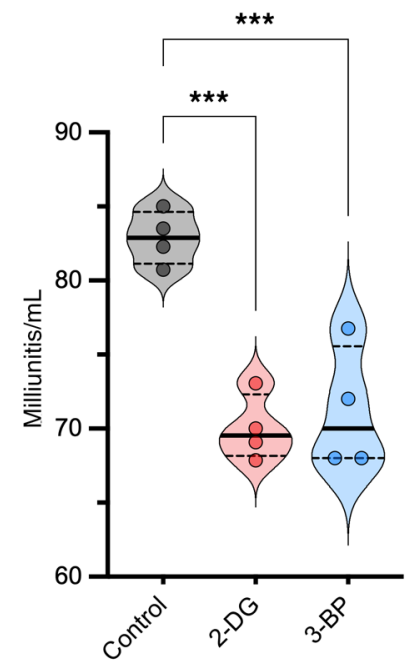

G

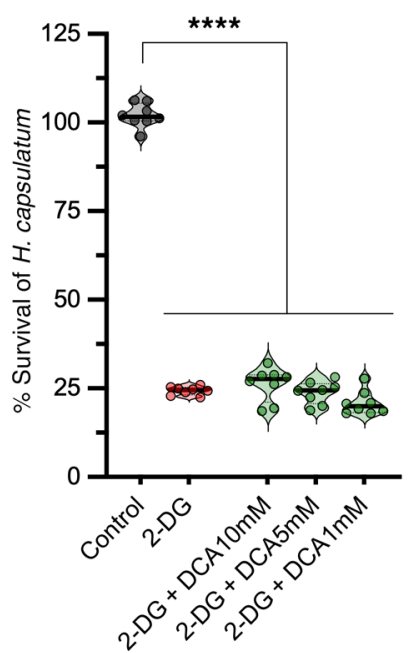

B

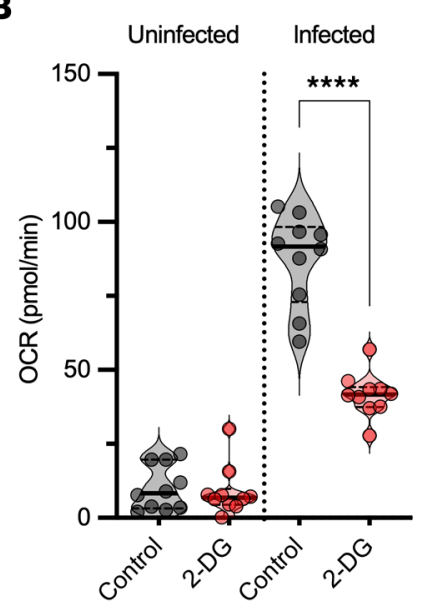

E

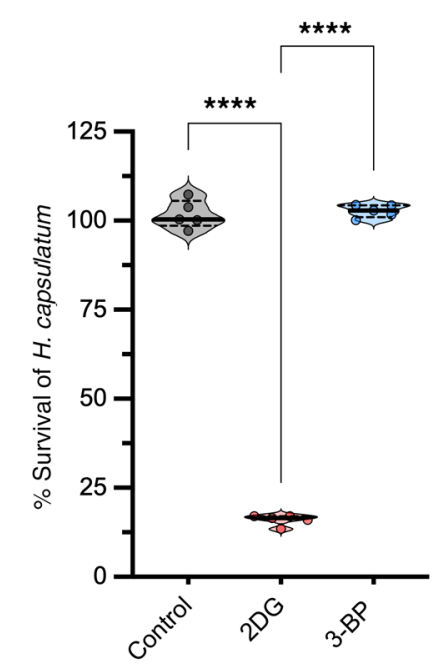

H

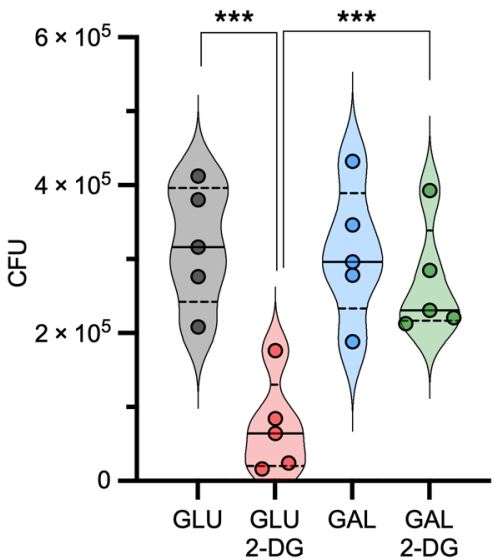

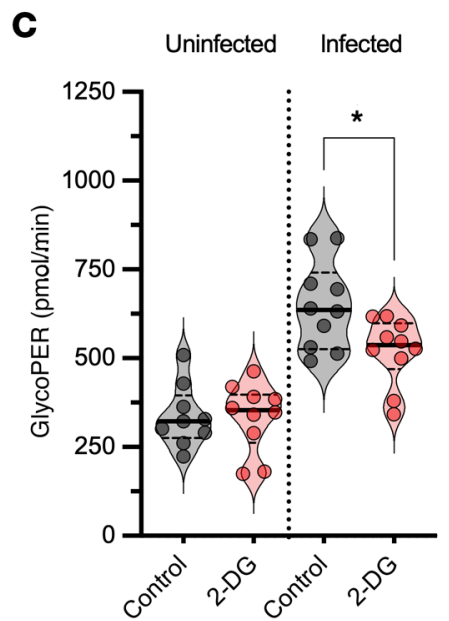

$\mathbf{F}$

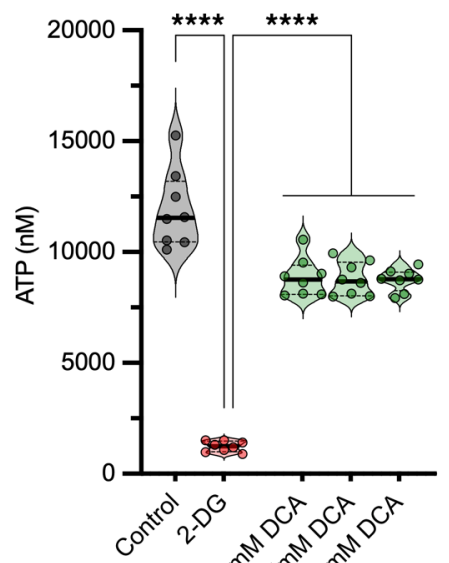

$\Omega_{0} 0^{x}, 0^{\circ}, 0^{x}$

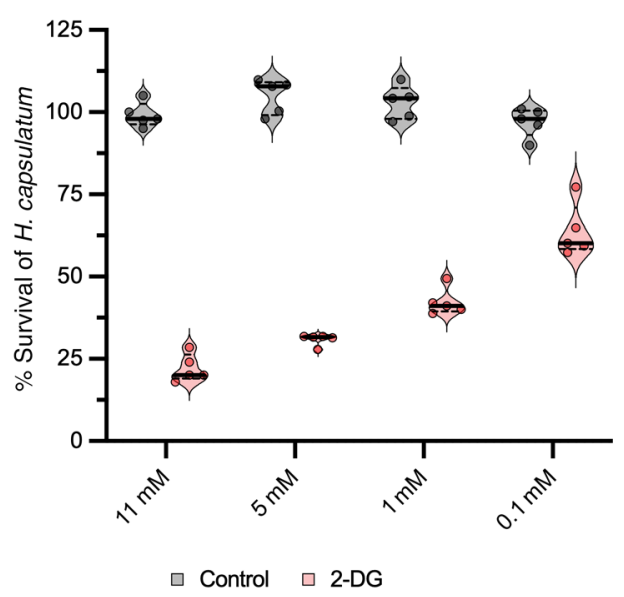


Figure 4. ATP is not required for 2-DG activity. (A) ATP measurement. Two-tailed $t$ test. (B) Basal mitochondrial oxygen consumption rate (OCR). Two-tailed $t$ test. (C) Basal glycolytic proton efflux rate (glycoPER). Twotailed $t$ test. (D) HK activity of infected BMDMs treated with $5 \mathrm{mM} 2-\mathrm{DC}$ or $10 \mu \mathrm{M}$ 3-BP. One-way ANOVA with Tukey's multiple-comparison test. (E) XTT assay from infected BMDMs treated with $5 \mathrm{mM} 2-\mathrm{DG}$ or $10 \mu \mathrm{M}$ 3-BP. One-way ANOVA with Tukey's multiple-comparison test. (F) ATP measurement from infected BMDMs treated with $5 \mathrm{mM}$ 2-DG and DCA. One-way ANOVA with Tukey's multiple-comparison test. (C) XTT assay from infected BMDMs treated with 5 mM 2-DG and DCA. One-way ANOVA with Tukey's multiple-comparison test. (H) CFU assay from infected BMDMs treated with $5 \mathrm{mM} 2-\mathrm{DG}$ in the presence or not of $11 \mathrm{mM}$ glucose or $11 \mathrm{mM}$ galactose. One-way ANOVA with Tukey's multiple-comparison test. (I) XTT assay from infected BMDMs treated with $5 \mathrm{mM}$ 2-DG in the presence of several concentrations of glucose. One-way ANOVA with Tukey's multiple-comparison test. BMDMs were infected with MOI 1:5 (A-I) treated with $5 \mathrm{mM}$ 2-DG for 6 hours (A-D and $\mathbf{F}$ ) and for 24 hours (E and $\mathbf{G}$ ). Data are representative of 5 (A, E, $\mathbf{H}$, and $\mathbf{I}), 10$ (B and $\mathbf{C}$ ), 4 (D), or 8 ( $\mathbf{F}$ and $\mathbf{G}$ ) independent experiments. Violin plots show the median (line) and quartiles (dashed line). ${ }^{*} P<0.05 ;{ }^{* *} P<0.001 ;{ }^{* * * *} P<0.0001$.

2-DG-mediated killing of $H$. capsulatum did not rely on modification of LAP (Figure 5C).

2-DG not only causes energy stress as a glycolysis inhibitor but also interferes with $\mathrm{N}$-linked glycosylation, resulting in ER stress (14). Since autophagy and LAP were dispensable, we focused on whether ER stress contributes to the antifungal activity exerted by 2-DG in an autophagy-independent manner. First, we sought to confirm that 2-DG produced ER stress in macrophages via Western blot and bulk RNA-Seq on infected and uninfected BMDMs. For Western blot, we examined a major ER chaperone protein GRP78 (BIP) and the transcription factor 4 (ATF-4) since these are increased in ER stress (31, 32). 2-DG enhanced expression of BIP and ATF4 (Figure 5D). Analysis of bulk RNA-Seq data revealed that a number of genes in the ER stress pathway were upregulated, thus supporting the assertion that 2-DG provokes ER stress (Figure $5 \mathrm{E}$ ). We inquired whether another ER stressor, tunicamycin, mimicked the effect of 2-DG. Treatment with tunicamycin did not reduce $H$. capsulatum survival (Figure 5F). We validated that ER stress was not contributing to the killing by treating macrophages with mannose, which relieves ER stress caused by 2-DG (33). Mannose addition to infected BMDMs reduced ER stress as assessed by the quantity of BIP and ATF-4 (Figure 5D) but did not reverse $H$. capsulatum killing provoked by 2-DG (Figure $5 G$ ). Taken together, ER stress caused by 2-DG did not cause the killing of yeast cells by $2-D G$.

2-DG impairs the import of zinc in BMDMs. Since ER stress and autophagy did not explain the mechanism by which 2-DG acted, we probed the possibility that this glucose analog triggered nutritional immunity. Zinc (13), iron (34-36), and copper (6) are vital for survival of $H$. capsulatum; hence, we investigated whether 2-DG changed the amount of these metals in BMDMs and yeast cells. We quantified the metal content in H. capsulatum and BMDMs by inductively coupled mass spectrometry (ICP-MS). H. capsulatum from 2-DG-treated BMDMs exhibited reduced zinc but increased iron, whereas copper quantities were unchanged (Figure 6A). Since 2-DG manipulated the metal content in yeast cells, we ascertained whether this result was correlated with an alteration of trace metal in host cells. 2-DG decreased cytoplasmic zinc and iron, but not copper, in uninfected and infected BMDMs (Figure 6B). To support the data obtained with ICP-MS, we utilized zinpyr-1 in combination with flow cytometry to assess zinc content in uninfected BMDMs. Although zinpyr-1 was not able to discriminate between bound and free or labile zinc, 2-DG treatment decreased the MFI of zinpyr-1 in macrophages (Figure 6C).

Using size-exclusion chromatography ICP-MS (SEC-ICP-MS), fractions that elute between 10 and 17.5 minutes correspond to higher molecular weight moieties. Those found between 17.5 and 22.5 contain metallothioneins (MTs), and fractions between 25 and 30 minutes contain labile zinc, which is the pool that is readily available for use by $H$. capsulatum (13). We used this technique to ascertain whether the decrement in zinc resulted in less labile zinc. 2-DG-treated BMDMs exhibited considerably less labile zinc (Figure 6D). Thus, 2-DG affected total and labile zinc, thereby depriving the fungus of an essential element.

We hypothesized that 2-DG modified zinc distribution in BMDMs by manipulating zinc transporters. To test this assertion, gene expression of zinc importers (ZIPs; Slc39a1-14) and exporters (ZNTs; Slc30a1-10) was analyzed. Slc39a2 (ZIP2) and Slc30a1 (ZNT1) were upregulated in infected 2-DG-exposed BMDMs by 8 -fold and 1.7-fold, respectively (Figure 6D). To determine the influence of these transporters on fungal killing, we silenced Slc39a2 and Slc30a1 (Supplemental Figure 3A). Reducing the expression of Slc39a2 and Slc30a1 did not reverse the effect of 2-DG (Figure 6E). Slc39a1 (ZIP1) was diminished by 2-fold in infected BMDMs that received 2-DG (Figure 6F). We explored whether downregulation of Slc39a1 was associated with fungal killing; we silenced this transporter in infected cells (Supplemental Figure 3B). Infected BMDMs deficient in Slc39a1 exhibited similar fungal viability compared with the controls (Figure 6G). Our results revealed that although 2-DG decreased the quantity of zinc in macrophages and yeast cells, the outcome was not a consequence of changes in the expression of these particular zinc transporters.

Since alteration of individual importers or exporters did not reverse the killing effect of 2-DG, we theorized that this chemical compromised the global import of zinc. Zinc has 5 stable isotopes, ${ }^{64} \mathrm{Zn},{ }^{66} \mathrm{Zn},{ }^{67} \mathrm{Zn},{ }^{68} \mathrm{Zn}$, and ${ }^{70} \mathrm{Zn}$, with natural abundances of $49.2 \%$, $27.8 \%, 4.0 \%, 18.4 \%$, and $0.6 \%$, respectively. We incubated macrophages in medium containing only ${ }^{68} \mathrm{Zn}$ and exposed cells to $2-\mathrm{DG}$ or vehicle. Zinc influx was monitored by alterations of ${ }^{68} \mathrm{Zn} /{ }^{64} \mathrm{Zn}$ ratio. If 2-DG hampered import, we expected to detect a lower ratio compared with the control. Indeed, 2-DG-exposed BMDMs displayed less zinc import (Figure 6H). The decrement caused by $2-D G$ was principally in the higher molecular weight fractions and not in the free zinc. Collectively, 2-DG impeded zinc import, thereby reducing zinc, especially the amount of free zinc.

The reduction of zinc leads to the death of $H$. capsulatum. To prove that zinc deficiency affected yeast cell survival, we added either $\mathrm{ZnSO}_{4}$ or $\mathrm{MgSO}_{4}$ simultaneously with 2-DG to cultures of BMDMs harboring yeast cells. 2-DG-treated BMDMs supplemented with exogenous $\mathrm{ZnSO}_{4}$ but not $\mathrm{MgSO}_{4}$ failed to eliminate $H$. capsulatum in a dose-dependent manner (Figure 7A). Adding zinc to macrophages may exert its effect by altering phagocyte function independent of restoring this metal to the fungus. To support the hypothesis that the effect of zinc supplementation for- 
A
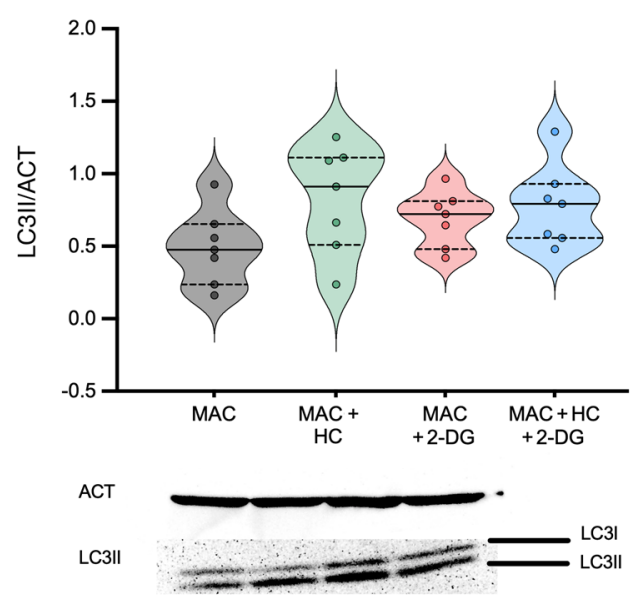

B
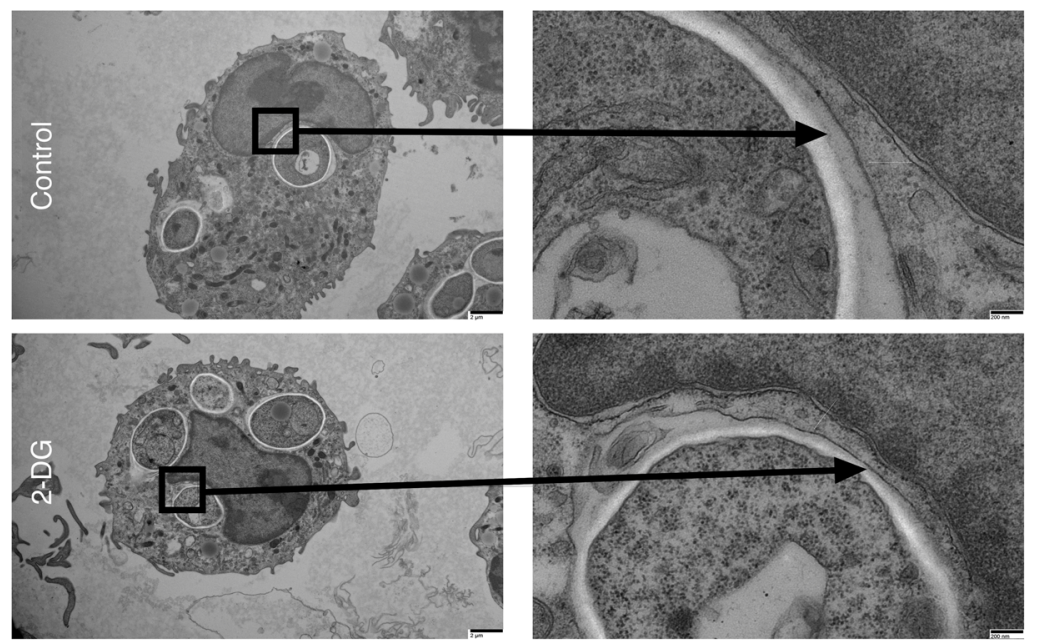

C

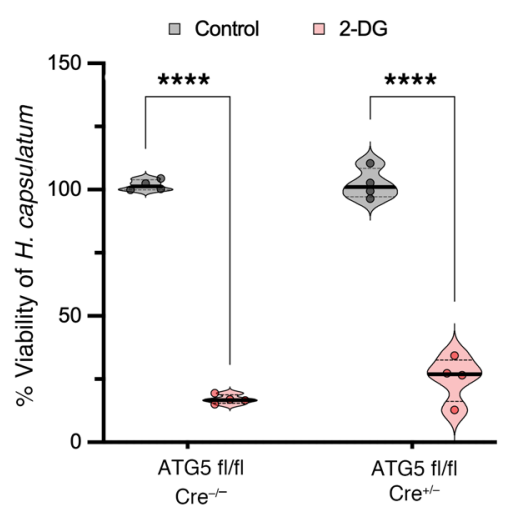

E

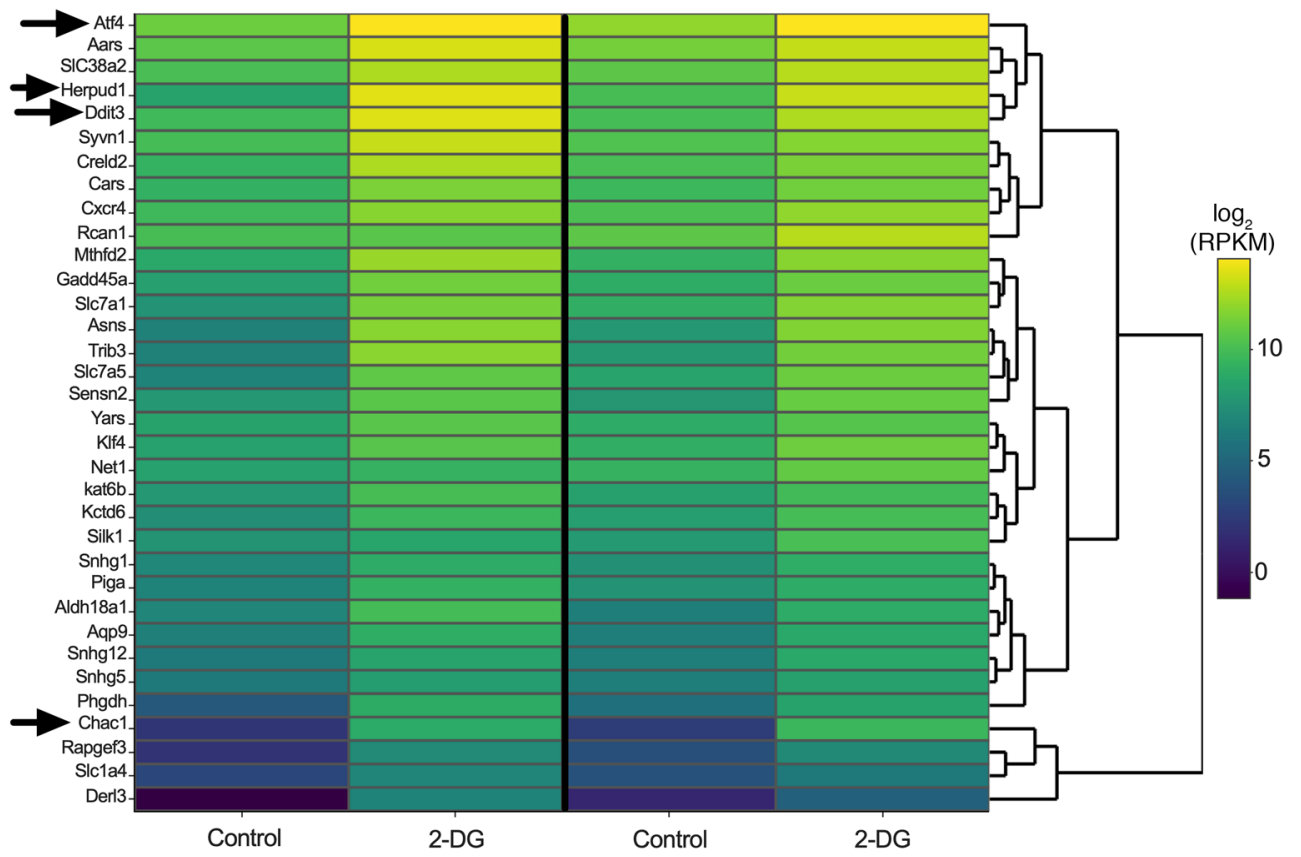

D
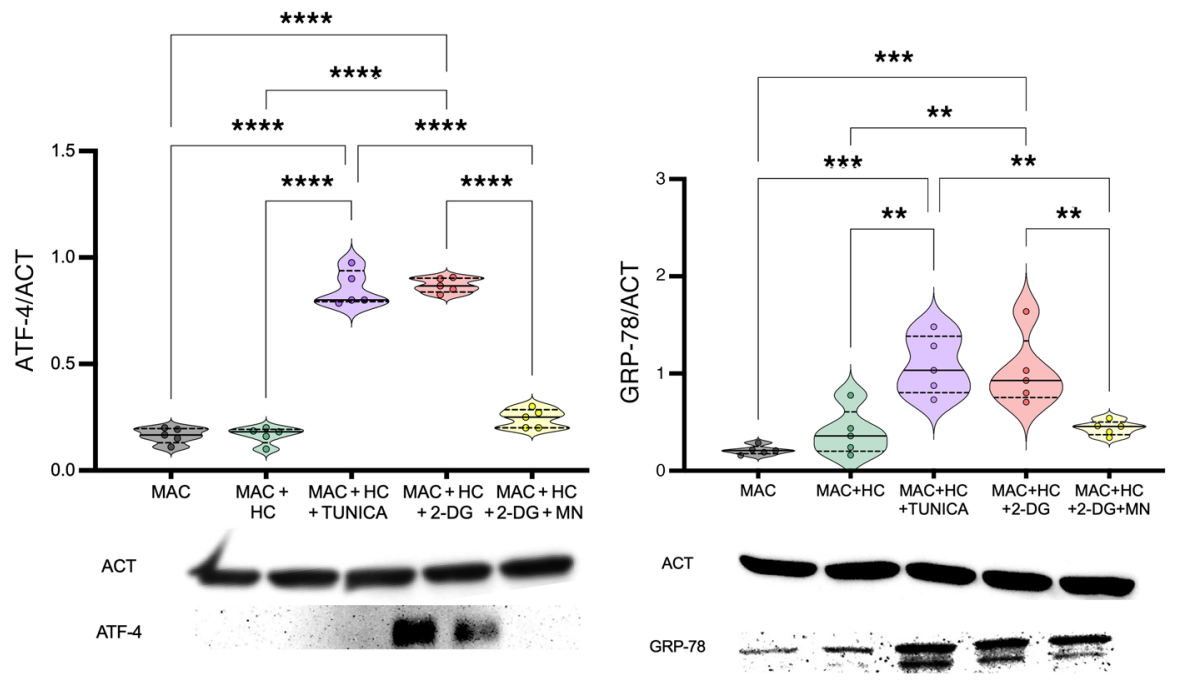

F

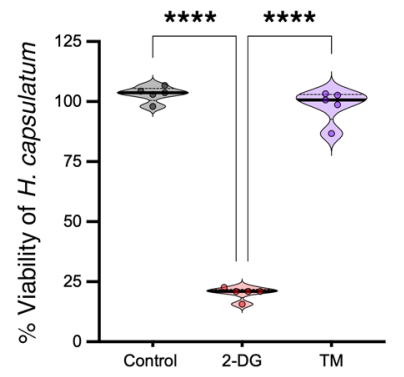

G

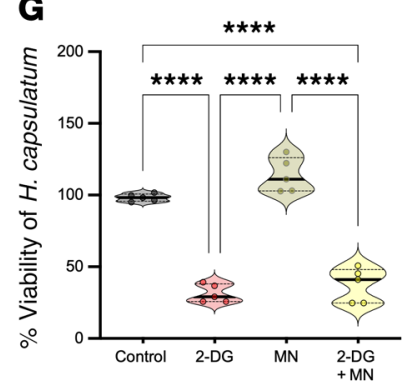


Figure 5. Autophagy and ER stress are not required for the effect of 2-DC. (A) LC3-I and -II immunoblot. One-way ANOVA with Tukey's multiplecomparison test. MAC, BDMCs; HC, H. capsulatum. (B) Transmission electron microscopy. Original magnification, $\times 2000$ (left) and $\times 8000$. The arrows indicate the membrane surrounding the $H$. capsulatum compartment. (C) XTT assay. Two-way ANOVA with Sidak's multiple-comparison test. (D) GRP78 and ATF-4 immunoblot from BMDMs; 5 mM mannose and $2.5 \mu \mathrm{g} / \mathrm{mL}$ tunicamycin. One-way ANOVA with Tukey's multiple-comparison test. (E) RNA-Seq analysis from BMDMs after 3 hours of 2-DC treatment. RPKM, reads per kilobase of transcript, per million mapped reads. Arrows indicate ER stress genes. (F) XTT assay from infected BMDMs treated with $5 \mathrm{mM} 2$-DC and $2.5 \mu \mathrm{g} / \mathrm{mL}$ tunicamycin. One-way ANOVA with Tukey's multiple-comparison test. (C) XTT assay, 5 mM mannose. One-way ANOVA with Tukey's multiple-comparison test. BMDMs were infected with MOI 1:5 (A-F) treated with $5 \mathrm{mM}$ 2-DG for 6 hours (A, B, and $\mathbf{D}), 3$ hours (E), or 24 hours (C, F, and $\mathbf{G})$. Data are representative of 4 to 7 (A and $\mathbf{D}$ ) or 3 (B, $\mathbf{C}, \mathbf{F}$, and $\mathbf{G}$ ) independent experiments. Violin plots show the median (line) and quartiles (dashed line). ${ }^{* *} P<0.01 ;{ }^{* *} P<0.001 ;{ }^{* * *} P<0.0001$.

tified the organism and did not disarm macrophages, we precultured H. capsulatum with zinc (Supplemental Figure 3A) and fed these organisms to BMDMs in the presence of 2-DG. Zinc-loaded H. capsulatum survived 2-DG treatment (Figure 7B). These data indicate that 2-DG manipulates zinc availability to promote killing of $H$. capsulatum.

Given that H. capsulatum isolated from 2-DG-treated BMDMs exhibited increased iron, we investigated the impact of iron on $H$. capsulatum viability. After 24 hours of incubation in iron-supplemented medium, yeasts manifested similar viability as controls (Supplemental Figure 3B). Likewise, addition of exogenous iron to medium did not alter viability of yeasts residing in BMDMs (Figure 7A). Yeast cells preloaded with iron and incubated with BMDMs exhibited similar viability as control yeast cells (Figure 7B and Supplemental Figure 3A). We conclude that iron did not contribute to the effect of 2-DG.

MTs are cysteine-rich proteins that bind up to 7 zinc ions and donate 1 ion (37). Enhanced expression of these molecules induced by GM-CSF sequesters this metal from the fungus (13). We considered the possibility that the effect of 2-DG was dependent on MTs. This metabolic inhibitor did not modify expression of MTs (Supplemental Figure 4B). Moreover, BMDMs lacking MTs killed H. capsulatum upon exposure to 2-DG (Figure 7C). Taken together, MTs were dispensable for killing by 2-DG.

We probed the postulate that the unavailability of zinc would result in changes in the expression of zinc transporters in ingested yeast cells. After treatment with 2-DG for 6 hours, expression of the high-affinity zinc importer HcZRT2 and the zinc-dependent transcriptional regulator HcZAP1 were significantly decreased (Figure 7C). The low-affinity zinc importer HcZRT1 remained unchanged. A putative vacuolar exporter, HcZRT3, which would presumably provide zinc to the yeast cytosol, increased upon exposure to 2-DG (Figure 7C). Since iron content in yeast cells was elevated after exposure to 2-DG, we examined iron transporters. $H c S I D 1, H c S I D 3$, and HcMFS1 were significantly decreased at 6 hours after addition of 2-DG (Supplemental Figure 4C).

Since 2-DG treatment impaired zinc import in the macrophage leading to a zinc-deprived microenvironment, we investigated whether 2-DG has a direct action on Histoplasma yeasts. To mimic the acidic, nutrient-limited, and zinc-free environment, we used RPMI with and without zinc at pH 7.2 and 6.5. We chose RPMI because it contains fewer nutrients than other media that are used to grow Histoplasma yeasts. We also asked whether 2-DG6P had a direct impact on Histoplasma yeasts in this microenvironment. After 24 hours of incubation, neither 2-DG nor 2DG6P impaired fungal viability in a zinc-free environment. The acidic $\mathrm{pH}$ did not promote the ability of 2-DG or 2-DG6P to reduce viability of the Histoplasma yeasts (Figure 7E).

\section{Discussion}

In this study, we have unequivocally established that the glycolysis inhibitor 2-DG arms mouse and human macrophages to express potent antifungal activity. 2-DG-treated BMDMs eliminated approximately $75 \%$ of Histoplasma yeasts in vitro after 24 hours of treatment. A 2- to 3-fold difference in growth was observed between 24 and 48 hours. Presumably, this growth was a result of yeasts that escaped killing. Since the in vitro half-life of 2-DG is unknown, it is difficult to know whether the potency of 2-DG requires additional exposure to the compound to fully eradicate yeast cells. In vivo, this metabolic inhibitor substantially reduced fungal burden. Mechanistically, the effect of 2-DG in macrophages did not depend on a change in host glycolysis; rather this compound unexpectedly diminished the quantity of zinc in the cytosol and in yeast cells residing in phagosomes. Since this trace metal is vital for fungal survival (13), the net result was death of yeast cells by zinc starvation. Deficient import was the root cause of zinc reduction, but this defect could not be localized to a single transporter. Operationally, the impaired zinc ingress encompassed dysfunction of at least 2, if not all, importers. This conclusion is based on the fact that various ZIPs shuttle zinc in disparate ways (38). At present, the methodology to quantify metal import only permits analysis of total zinc and not the ingress of a single zinc ion. The latter would be exceptionally useful to understand how 2-DG impedes the transport of an individual ion. Nevertheless, the data revealed that the activity of 2-DG extended far beyond manipulating glycolysis.

We verified that zinc deficiency promoted by 2-DG greatly diminished yeast cell viability using 2 approaches: supplementation of medium with $\mathrm{ZnSO}_{4}$ and fortification of yeast cells with zinc; both of which reversed killing. The latter approach enabled us to circumvent the possibility that zinc directly modulated macrophage behavior. The outcome appears to be selective for 2-DG and its analogs (2-FDG and 2-FDM) since 3-bromopyruvate, which has the same target, did not reduce fungal survival. The killing activity of 2-DG did not depend on a change in ATP production. Although this chemical diminished the amount of ATP generated by macrophages, restoring ATP production with DCA did not affect the lethality of 2-DG. The failure to import zinc was not a result of changes in ATP.

Frequently, 2-DG is used to document how glycolysis influences the responses and antimicrobial activity of macrophages after invasion by intracellular pathogens. The effect of this chemical appears to be somewhat stochastic and pathogen dependent. Both positive and negative outcomes have been reported. 2-DG undermines the ability of macrophages infected with $M$. tuberculosis to eliminate the pathogen either in vitro or in vivo $(39,40)$. 

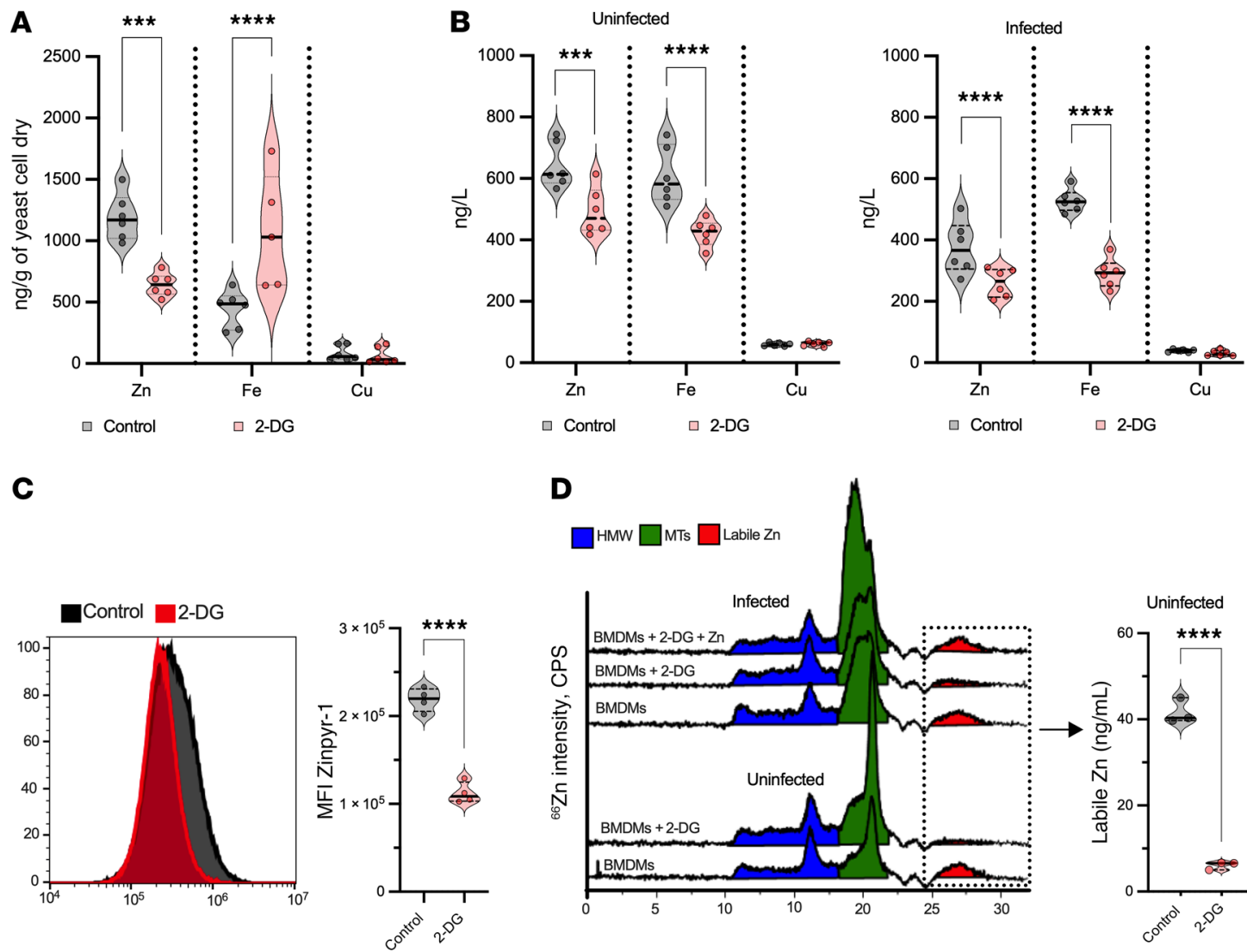

$\square$ Control

$\square$ 2-DG

$\square$ Control $\square$ 2-DG

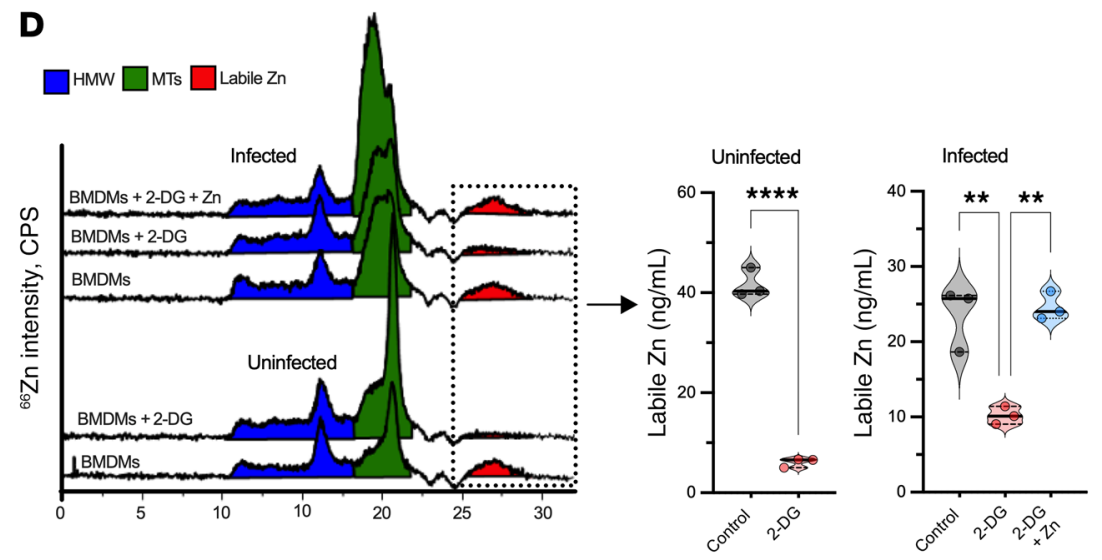

\section{E}

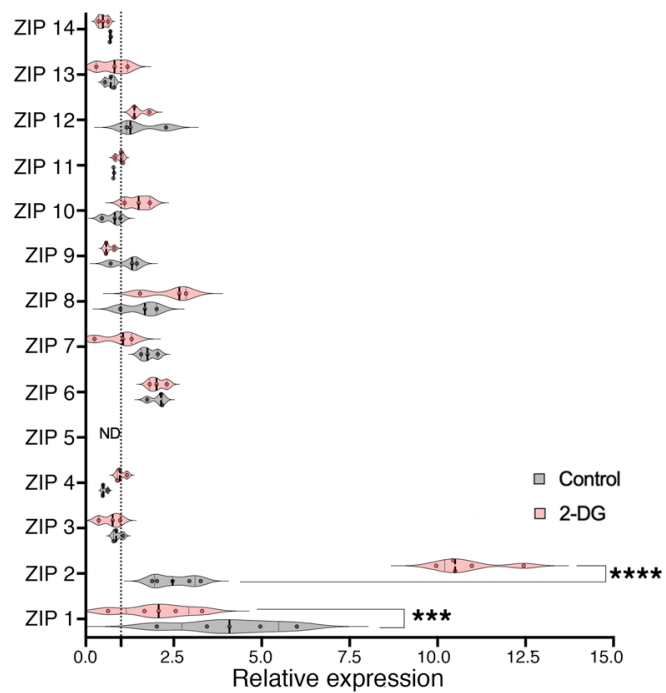

$\mathbf{F}$

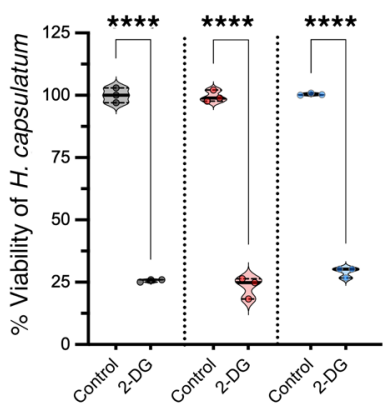

口 Scramble siRNA $\square$ Slc39a2 siRNA

Slc30a1 siRNA
G

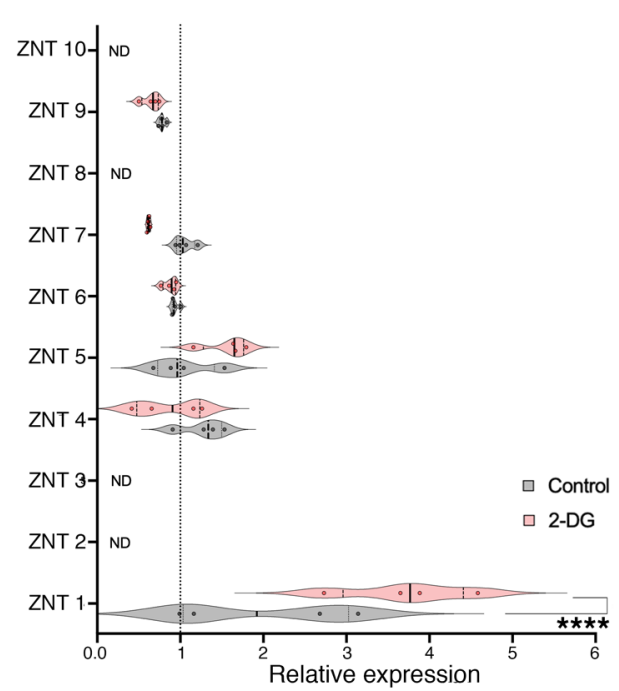

H

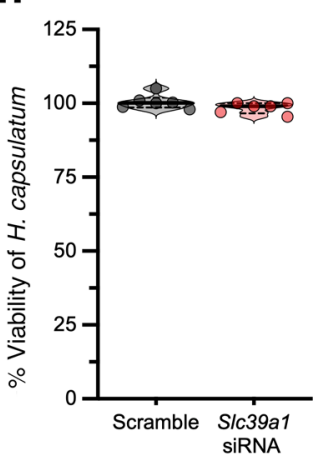

I

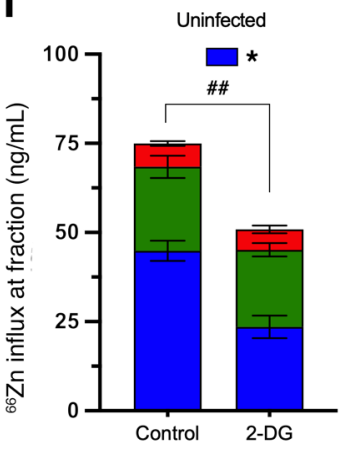

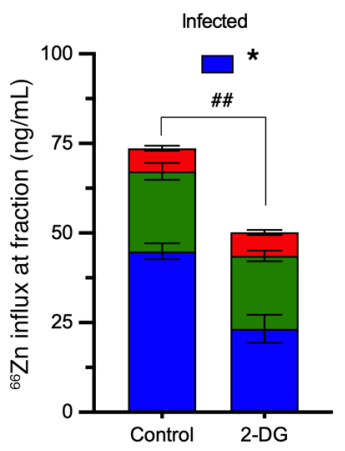

$\square$ HMW

$\square$ Labile Zn 
Figure 6. 2-DG compromises BMDM uptake of zinc. (A) Metal quantities from $H$. capsulatum that were within BMDMs treated with 2-DG for 6 hours. Two-tailed $t$ test. (B) Metal quantities from uninfected and infected BMDMs treated with 2-DG for 6 hours. Two-tailed $t$ test. (C) Flow cytometry of uninfected BMDMs stained with zinpyr-1 and treated with 2-DG. Two-tailed $t$ test. (D) Chromatogram from ICP-MS. Labile zinc from infected and uninfected BMDMs treated with 2-DG or 2-DG plus zinc supplementation $(250 \mu \mathrm{M})$. Not infected, 2-tailed $t$ test. Infected, one-way ANOVA with Tukey's multiple-comparison test. HMW, high molecular weight fraction. (E) Gene expression of zinc importers (ZIPs; SIc39a1-14) from infected BMDMs treated with 2-DG for 6 hours. Two-way ANOVA with Sidak's multiple-comparison test. The housekeeping gene and all data were normalized to uninfected BMDMs as reference (dotted line). (F) XTT assay from infected BMDMs lacking ZIP2 and ZNT1 treated with 2-DC for 24 hours. One-way ANOVA with Tukey's multiple-comparison test. (G) Gene expression of zinc exporters (ZNTs; SIc30a1-10) from infected BMDMs treated with 2-DG for 6 hours. Two-way ANOVA with Sidak's multiple-comparison test. The housekeeping gene and all data were normalized to uninfected BMDMs as reference (dotted line). (H) XTT assay from infected BMDMs lacking ZIP1. The assay was performed 24 hours after infection. Two-tailed $t$ test. (I) Distribution of exogenous ${ }^{68} \mathrm{Zn}$ taken up by BMDMs after 6 hours of 2-DC treatment. ${ }^{\# \#} P<0.01$ for differences between totals by 2 -tailed $t$ test. ${ }^{*} P<0.05$ for differences between HMW fractions by one-way ANOVA with Tukey's multiple-comparison test. BMDMs were infected with $\mathrm{MOI}$ 1:5 treated with $5 \mathrm{mM}$ 2-DG (A-I). Data are representative of 6 (A, B, and $\mathbf{H}), 3$ to $4(\mathbf{C}, \mathbf{D}$, and $\mathbf{F})$, or 3 (E and $\mathbf{G})$ independent experiments. Violin plots show the median (line) and quartiles (dashed line); bar graphs show mean \pm SEM. ${ }^{*} P<0.05 ;{ }^{* *} P<0.01$; ${ }^{* *} P<0.001$; ${ }^{* * * *} P<0.0001$

Likewise, 2-DG blunts the antimycobacterial activity of IFN- $\gamma$ activated macrophages (41). Although 2-DG promotes resistance to $L$. monocytogenes in vivo, the mechanism is not a result of arming BMDMs (42). Plasmodium berghei-infected mice administered 2-DG manifest enhanced survival as a consequence of changes in the formation of intracerebral microthrombi and hemorrhage and not alterations in parasite burden or degree of anemia (43). Conversely, 2-DG may benefit the host. Replication of murine norovirus in 2-DG-treated macrophages is suppressed because this compound decreases viral protein production and RNA (44). Similarly, a reduction in the number of L. pneumophila (10) or $B$. abortus (9) in human macrophages or the human macrophage-like line THP-1, respectively, was observed after administration of the compound. Bacterial killing was due to glycolysis inhibition.

One of the activities of 2-DG that is often overlooked is ER stress generation by interfering with $\mathrm{N}$-linked glycosylation (14). This process triggers the unfolded protein response (UPR), which is a complex network of intracellular signaling that reestablishes cellular homeostasis (45). Autophagy is stimulated as a consequence of the UPR (46). One outcome of autophagy is elimination of intracellular pathogens, called xenophagy (27). Accordingly, 2-DG promotes autophagy to spur macrophage killing of L. pneumophila (29). However, we found no evidence that autophagy was elevated or contributed to the action of 2-DG against $H$. capsulatum .

Nutritional immunity is another strategy that macrophages utilize to combat intracellular pathogens. Transition metals such as zinc (13), iron (34-36), and copper (6) are necessary for survival of multiple pathogens, including $H$. capsulatum, within macrophages. Zinc is an essential nutrient for $H$. capsulatum growth, and merely restricting access to this trace metal results in the death of yeast cells (47). However, the decrease in the viability of Histoplasma yeasts in zinc-free media was not as robust as within macrophages. It is difficult, if not impossible, to recreate the microenvironment in the phagolysosome. This organelle contains many degradative enzymes, including various cathepsins, proteases, lysozymes, and lipase in unknown concentrations (48). Moreover, the intracellular contents of the phagolysosome are a much more inhospitable milieu than the zinc-deprived media. Many of these enzymes, particularly cathepsins, are less active in the presence of zinc $(49,50)$. Hence, it is conceivable that the deprivation in zinc in the phagolysosome of 2-DG-treated cells enhances the activity of these degradative enzymes on yeast cells unable to cope with this attack (51).

One way to deny metals is to sequester them from the pathogen. This strategy is operative in H. capsulatum-infected macrophages exposed to GM-CSF. In this scenario, total macrophage zinc is increased but labile zinc is low because MTs 1 and 2 bind the metal and deplete the labile pool (13). In contrast, 2-DG did not produce MT-dependent sequestration. Rather, this metabolic inhibitor decreased the amount of total and free zinc in macrophages, leading to a decrease in yeast cells. Analysis of zinc import using ${ }^{68} \mathrm{Zn}$ revealed diminished entry of this metal into macrophages. By SEC-ICP-MS, the principal decrease was apparent in the higher molecular weight fractions and not in the labile pool. This finding strongly suggests that macrophages endeavor to replace the labile pool, which is instrumental for the biological function of enzymes and transcription factors. We entertained the possibility that upregulation of the zinc transporters affected changes in the metal content and produced yeast cell death, but that postulate was not verified. The upregulation in Zip2, in particular, can be interpreted as a response to sensing low zinc in macrophages. We conclude that 2-DG triggered a comprehensive dysfunction in zinc importers.

Analysis of the $H$. capsulatum zinc transporters revealed an interesting pattern. Despite the paucity of zinc in yeast cells, the major genes involved in import $\mathrm{HcZrt1}$ and $\mathrm{HcZrt} 2$ were unchanged or depressed, respectively. Likewise, the metal-responsive transcriptional regulator HcZap3 was downregulated. The expectation was that these genes would be upregulated to counteract the decrement of available zinc to the fungus. The finding that all 3 were not increased in expression implies that the fungus, although viable at this time, was inappropriately responding to environmental changes. This finding may signify that the fungus does not cope well with zinc deprivation.

Macrophages exposed to 2-DG exhibited less iron in the cytosol, whereas the content of this metal in ingested $H$. capsulatum revealed less zinc but more iron when compared with yeast cells in control macrophages. This increase in iron likely accounts for the decreased expression of iron transporters. These results indicate that yeast cells exhibit a preference for iron over zinc when confronted with an external deficiency in both.

2-DG is chemically similar to glucose; the molecular difference between them is only a single oxygen molecule. Herein, we demonstrated that this small modification imposed an impact on glucose metabolism and on the host pathogen relationship. While glucose is needed to fuel glycolysis, 2-DG is 
A

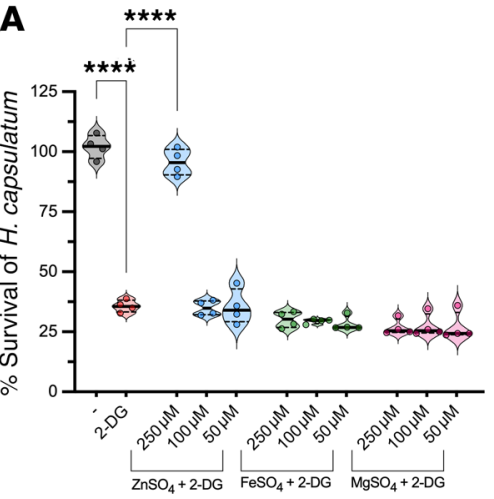

D

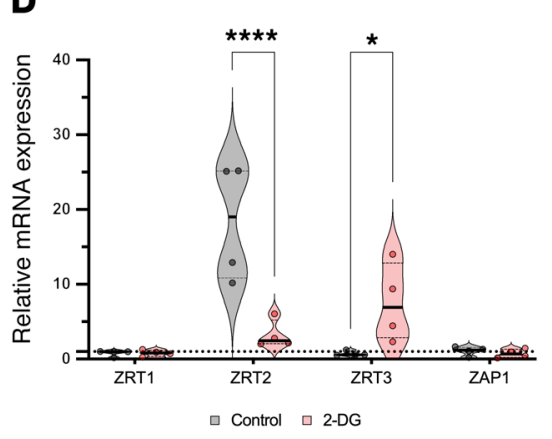

B

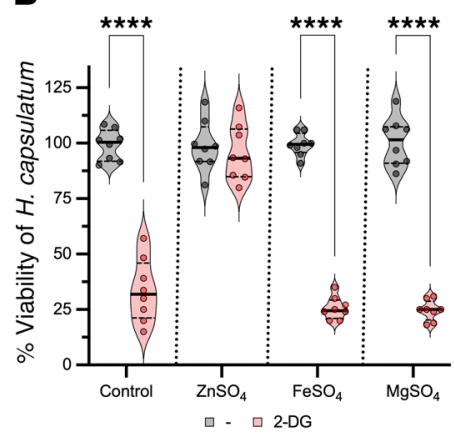

C

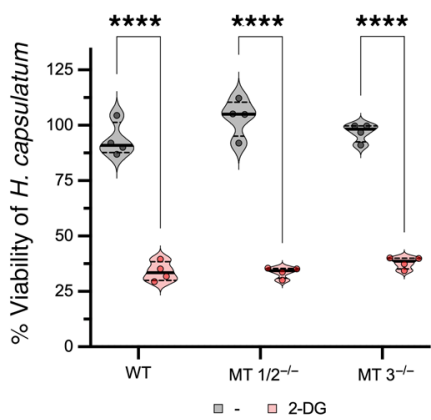

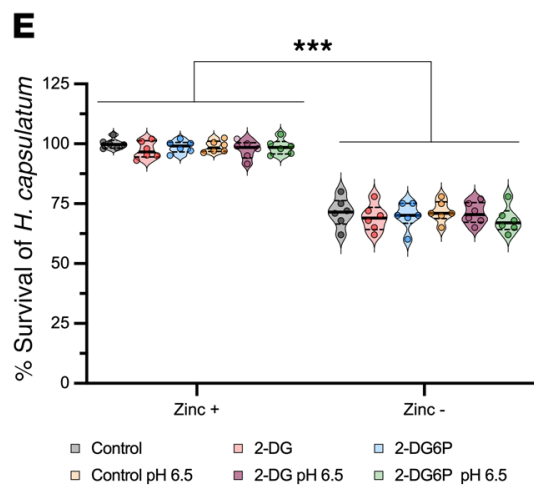

Figure 7. Zinc deficit results in $\boldsymbol{H}$. capsulatum death. (A) XTT assay from infected BMDMs treated with 2-DG with or without metal supplementation for 24 hours. One-way ANOVA with Tukey's multiple-comparison test. (B) XTT assay from BMDMs infected with metal-loaded $H$. capsulatum treated with 2-DG for 24 hours. Two-tailed $t$ test. (C) XTT assay from BMDMs lacking MT1/2 and MT3 treated with 2-DG for 24 hours. One-way ANOVA with Tukey's multiple-comparison test. (D) Cene expression of zinc transporters from H. capsulatum within macrophages treated with 2-DG for 6 hours. Two-way ANOVA with Sidak's multiple-comparison test. The housekeeping gene and all data were normalized to $\mathrm{H}$. capsulatum-only as reference (dotted line). (E) XTT from H. capsulatum treated with $5 \mathrm{mM}$ 2-DG, $5 \mathrm{mM}$ 2-DG6P with zinc-deprived media, and in different pH. One-way ANOVA with Tukey's multiple-comparison test. BMDMs were infected with MOI 1:5 treated with $5 \mathrm{mM}$ 2-DC (A-D). Data are representative of $4(\mathbf{A}, \mathbf{C}$, and $\mathbf{D}), 8(\mathbf{B})$, or 6 (E) independent experiments. Violin plots show the median (line) and quartiles (dashed line). ${ }^{*} P<0.05$; ${ }^{* * *} P<0.001 ;{ }^{* * *} P<0.0001$.

commonly used to mimic glucose starvation. In addition, 2-DG competes with mannose in the growing lipid-linked oligosaccharide chain during the initial steps of $\mathrm{N}$-linked glycosylation. Displacement of mannose results in misfolded proteins causing ER stress that could lead to autophagy. These properties of 2-DG are well known. In contrast, our data revealed an unanticipated effect of this compound that seemingly has little connection to glycolysis. The finding that this compound manipulates zinc homeostasis in uninfected or H. capsulatum-infected macrophages expands the complexity of 2-DG activities. One consequence is the death of this fungus, which cannot overcome the scarcity of zinc. This finding may clarify how 2-DG restricts the intracellular growth of other pathogens. In the past, 2-DG has been evaluated in several clinical studies as an anticancer agent, mainly for its glycolysis-inhibiting property. Thus, the utility of this agent may be adjunctive therapy for histoplasmosis or malignancy may be a consequence not only of glycolysis but also zinc deprivation.

\section{Methods}

Mice. C57BL/6 mice were purchased from The Jackson Laboratory (stock number 000664). Animals were housed in isolator cages and maintained by the Department of Laboratory Animal Medicine,
University of Cincinnati, which is accredited by the Association for Assessment and Accreditation of Laboratory Animal Care. Bone marrow from ATG5 conditional knockouts and their controls were obtained from the NIH with permission from Noburo Mizushima, University of Tokyo.

Culture of H. capsulatum. H. capsulatum yeast cells (strain G217B) were grown at $37^{\circ} \mathrm{C}$ in Ham's F-12 medium (Sigma-Aldrich) supplemented with glucose $(18.2 \mathrm{~g} / \mathrm{L})$, glutamic acid $(1 \mathrm{~g} / \mathrm{L})$, HEPES $(6 \mathrm{~g} / \mathrm{L})$, and cysteine $(8.4 \mathrm{mg} / \mathrm{L})$ for 72 hours. Yeast cells were washed 3 times with HBSS and counted. For metal loading, yeasts were cultured as described above with the addition of metal supplementation (1 mM).

Generation of BMDMs. BMDMs were prepared by GM-CSF or M-CSF (BioLegend) (10 ng/mL) differentiation in RPMI media (Cytiva) containing 10\% FBS (Corning), gentamycin sulfate $(10 \mu \mathrm{g} / \mathrm{L})$, and $50 \mu \mathrm{M}$ 2-mercaptoethanol. GM-CSF cultures were fed on days 0 and 3 and M-CSF cultures were fed on days 0,2 , and 4 with media and cytokine. After 7 days of differentiation at $37^{\circ} \mathrm{C}$ and $5 \% \mathrm{CO}_{2}$, macrophages were harvested by trypsinization.

Generation of human macrophages. Human PBMCs were isolated from buffy coats (purchased from the Hoxworth Blood Center) of deidentified healthy blood by passage over a Ficoll layer for lymphocyte separation. Monocytes were then isolated by adherence in RPMI 1640 supplemented with $10 \%$ FBS, $50 \mu \mathrm{M}$ 2-mercaptoethanol, and 10 
mM HEPES using $175 \mathrm{~cm}^{2}$ plastic flasks. Cells were differentiated into MDMs by adding $10 \mathrm{ng} / \mathrm{mL} \mathrm{M-CSF}$ for 3 days at $37^{\circ} \mathrm{C}, 5 \% \mathrm{CO}_{2}$.

Isolation of primary human alveolar macrophages. Alveolar macrophages were obtained from bronchoalveolar lavage fluids of patients suspected of having lung masses or cough at the Department of Pulmonology, Universitätklinikum Schleswig-Holstein, Lübeck, Germany. Cells were washed 3 times in PBS and resuspended in RPMI 1640 supplemented with $10 \%$ heat-inactivated FBS (PAN Biotech), $50 \mu \mathrm{M}$ 2-mercaptoethanol, $2 \mathrm{mM}$ glutamine (Lonza), $10 \mathrm{mM}$ HEPES (Gibco), and $0.1 \%$ penicillin-streptomycin. Cells were spread in a 48 -well plate at a density of $0.5 \times 10^{6}$ cells/well. Nonadherent cells were washed off after 2 hours, and alveolar macrophages were incubated overnight before they were infected.

Survival assay. For evaluation of $H$. capsulatum survival, $2 \times 10^{5}$ BMDMs were seeded in a final volume of $100 \mu \mathrm{L}$ into a 96-well plate. After 24 hours, BMDMs were infected with $H$. capsulatum at different MOIs and treated with several chemical compounds. To quantify CFUs, BMDMs were lysed with $100 \mu \mathrm{L}$ of water for 30 minutes. Then, $100 \mu \mathrm{L}$ was seeded into Mycosel agar plates supplemented with agarose $(8 \mathrm{~g} / \mathrm{L})$, dextrose $(10 \mathrm{~g} / \mathrm{L})$, cysteine $(100 \mathrm{mg} / \mathrm{L})$, and defibrinated sheep blood $(50 \mathrm{~mL} / \mathrm{L})$. Plates were incubated at $37^{\circ} \mathrm{C}$ for 7 days and fungal colonies were enumerated. For the XTT assay, BMDMs were lysed with $50 \mu \mathrm{L}$ of water for 30 minutes. Then, $100 \mu \mathrm{L}$ of XTT solution was added to the well for 3 hours at $37^{\circ} \mathrm{C}$, at which point the absorbance was measured at $490 \mathrm{~nm}$. For XTT solution, $100 \mu \mathrm{L}$ of menadione (Thermo Fisher Scientific) $(1.7 \mathrm{mg} / \mathrm{mL}$ in acetone) was added into 10 $\mathrm{mL}$ of XTT (Thermo Fisher Scientific) solution $(0.5 \mathrm{mg} / \mathrm{mL}$ in water).

To evaluate viability of BMDMs, $2 \times 10^{5}$ cells were seeded in a final volume of $100 \mu \mathrm{L}$ into a 96-well plate. After the treatment with various chemical compounds, the supernatant was removed and 100 $\mu \mathrm{L}$ of XTT solution was added for 2 hours. The absorbance was measured at $490 \mathrm{~nm}$.

Phagocytosis assay. We examined the influence of 2-DG on macrophage phagocytosis by a microplate fluorometric assay (52). Briefly, $1 \times 10^{6}$ BMDMs were seeded in a final volume of $100 \mu \mathrm{L}$ into a 96-well plate. After 24 hours, BMDMs were infected with calcofluor white-stained (CFW-stained) H. capsulatum at an MOI of 5. For staining $H$. capsulatum, $1 \times 10^{8}$ yeasts were washed and resuspended in $10 \mathrm{~mL}$ of CFW solution $(100 \mu \mathrm{g} / \mathrm{mL}$ in PBS) and labeled for 15 minutes at $37^{\circ} \mathrm{C}$. After 3 washes, the yeasts were added to the macrophages with or without $5 \mathrm{mM}$ 2-DG. After 2 hours, $100 \mu \mathrm{L}$ of $0.4 \%$ trypan blue was added to the well and the CFW fluorescence was measured. Excitation wavelength was $360 \mathrm{~nm}$ and emission wavelength was $465 \mathrm{~nm}$. The gain was set at 100, with integration start of $0 \mathrm{~ms}$ and integration time of $200 \mathrm{~ms}$. Results were recorded in arbitrary fluorescence units (FUs).

ROS assay. For ROS evaluation, BMDMs were seeded in a final volume of $100 \mu \mathrm{L}$ into a 96-well plate. After 24 hours, BMDMs were infected with $H$. capsulatum at an MOI of 5 and treated with $200 \mu \mathrm{M}$ apocynin (Sigma) for 1 hour. After that, $2.5 \mu \mathrm{L}$ of zymosan $(20 \mu \mathrm{g} / \mathrm{mL}$; Sigma-Aldrich) was added following the addition of $100 \mu \mathrm{L}$ of luminol solution (20 mM luminol + 0.5 U/mL HRP; Sigma-Aldrich). Immediately, the plate was submitted for chemiluminescence analysis in a plate reader for 1.5 hours at the desired temperature. The plate reader settings were gain $=120$ to 135 and integration time $=1.0$ seconds, and the minimum interval possible for reading in-plate reader measurements was generated.
Phagosomal pH measurements. BMDMs $\left(2 \times 10^{5}\right)$ were seeded in a final volume of $100 \mu \mathrm{L}$ into a 96-well plate. After 24 hours, BMDMs were infected with $H$. capsulatum at MOI of 5 and treated with $5 \mathrm{mM}$ 2-DG for 6 hours. The cells were stained with pHrodo Red AM Intracellular $\mathrm{pH}$ Indicator (Thermo Fisher Scientific) according to the manufacturer's instructions. Subsequent use of the Intracellular $\mathrm{pH}$ Calibration Buffer Kit (Thermo Fisher Scientific) allowed the intracellular $\mathrm{pH}$ to be quantified.

In vivo studies. Mice were i.n. infected with $2 \times 10^{6} \mathrm{H}$. capsulatum yeast cells in 30-50 $\mu \mathrm{L}$ volume. Mice were i.p. administered 10 $\mathrm{mg}(0.2 \mathrm{~mL})$ of 2-DG. Control animals were given an equal volume of HBSS. The mice were treated daily beginning on the day of infection and ceasing on the day before euthanasia. In another set of experiments, we began treatment on day 3 of infection and continued until the day before euthanasia.

For the CD4 and CD8 depletion, $300 \mu \mathrm{g}$ of anti-CD4 (clone GK1.5, BioLegend, 100402) and anti-CD8 (clone 2.43, BioLegend, 100702) were i.p. injected on days 7 and 3 and 0 of infection. As a control, we injected an equal amount of rat IgG2a (clone 1-1, Leinco Technologies, Inc., R1367) simultaneously. For neutrophil depletion, mice were i.p. injected with $0.1 \mathrm{mg}$ anti-Ly6G (clone 1A8, Bio X Cell, BE0075-1) or rat IgG2a the day before infection, day of infection, and 2 days after infection.

ATP extraction assay. BMDMs $\left(2 \times 10^{5}\right)$ were seeded into a 96-well plate. After 6 hours of 2-DG treatment, ATP were extracted with $30 \mu \mathrm{L}$ $1 \%$ trichloroacetic acid for 20 minutes on ice. The ATP determination kit was used to measure ATP (Thermo Fisher Scientific). The protocol used was done according to the manufacturer's instructions.

Zinpyr-1 staining and flow cytometry. After 6 hours of 2-DG treatment, BMDMs were stained with $5 \mu \mathrm{M}$ zinpyr-1 (Cayman Chemical) for 30 minutes. Subsequently, cells were stained with APC antimouse/human CD11b antibody (clone M1/70, BioLegend, 101211) and with 7-AAD (BioLegend). The samples were run in an Accuri C6 (BD Biosciences). Live CD11b+ BMDMs were analyzed using FlowJo software version 10.7 (BD Biosciences).

Metabolic function assay. BMDMs were seeded at $4 \times 10^{4}$ cells in $180 \mu \mathrm{L}$ complete RPMI per well in Seahorse XF 96-well culture microplates. Cells were allowed to adhere to the plate overnight at $37^{\circ} \mathrm{C}$ in $5 \% \mathrm{CO}_{2}$ before treatment and/or infection. On the day of assay, the cell culture medium was replaced with $180 \mu \mathrm{L}$ prewarmed, nonbuffered DMEM containing $10 \mathrm{mM}$ glucose, $2 \mathrm{mM}$ L-glutamine, and $1 \mathrm{mM}$ sodium pyruvate, followed by a 1-hour incubation at $37^{\circ} \mathrm{C}$ in ambient $\mathrm{CO}_{2}$. The basal glycolytic proton efflux rate (glycoPER) was measured according to the Seahorse XF Glycolytic Rate Assay Kit using a Seahorse XF96e analyzer. The glycolytic proton efflux rate was calculated via subtraction of nonglycolytic acidification (as measured after 2-DG treatment) from the total proton efflux rate.

OCR measurements were obtained for 55 minutes followed by sequential injections of oligomycin A $(1 \mathrm{mM})$, carbonyl cyanide 4 (trifluoromethoxy) phenylhydrazone (FCCP, $1.5 \mathrm{mM}$ ), and rotenone (200 $\mathrm{nM})$ plus antimycin $\mathrm{A}(4 \mathrm{mM})$ to measure OCR in response to mitochondrial stress. For ECAR, the glycolysis stress test was conducted by sequential addition of glucose $(10 \mathrm{mM})$, oligomycin $(10 \mathrm{mM})$, and 2-DG (50 mM).

SDS-PAGE and Western blotting. BMDMs $\left(1 \times 10^{6}\right)$ were seeded into a 24-well plate. After treatments with 2-DG, cells were lysed with $100 \mu \mathrm{L}$ of RIPA buffer (Sigma-Aldrich) supplemented with Halt 
Protease Inhibitor Cocktail (Thermo Fisher Scientific), and then resolved by SDS-PAGE. Total cell lysates were run in $4 \%$ to $20 \%$ Precise protein gels (Thermo Fisher Scientific) and transferred onto PVDF membranes. The immunoblot was prepared with LC3 B (1:500, Cell Signaling Technology, 2775S) and ATF-4 (1:1000, Cell Signaling Technology, 118145S) antibody and was probed with corresponding host-specific HRP-conjugated secondary antibodies and developed using SuperSignal West Femto Chemiluminescent Substrate (Thermo Fisher Scientific).

SEC-ICP-MS analysis and normalization of data. BMDMs $(2.5 \times$ $10^{6}$ ) were seeded into a 6-well plate. After treatments with 2-DG, cells were lysed with $0.1 \%$ SDS in water for 30 minutes on ice. Lysates were centrifuged and the $H$. capsulatum pellets and the supernatant were analyzed by SEC-ICP-MS. Fungal pellets were rinsed 3 times with cold PBS and transferred to a metal-free vial. Total metal analysis was performed by ICP-MS after acid mineralization. In brief, $100 \mu \mathrm{L}$ of $1: 1$ trace grade nitric acid/doubly deionized water and $20 \mu \mathrm{L}$ of $500 \mathrm{ppb}$ internal standard mixture were added to the pellet and heated on a dry bath for 30 minutes at $60^{\circ} \mathrm{C}$. The vials were vented, and the temperature was increased to $95^{\circ} \mathrm{C}$ for another 30 minutes. The samples were then cooled to room temperature and brought to a final volume of $1 \mathrm{~mL}$ with double deionized water.

Metal analysis was performed in an Agilent 7500ce ICP-MS system with a Scott double-pass spray chamber and Micromist nebulizer, a standard $2.5 \mathrm{~mL}$ torch, and nickel cones. The system was operated in collision mode with $3.5 \mathrm{~mL} / \mathrm{min}$ of $\mathrm{He}$ and a calibration range from 0.2 to $25 \mathrm{ppb}$ with the external calibration method. ${ }^{66} \mathrm{Zn},{ }^{63} \mathrm{Cu},{ }^{59} \mathrm{Co},{ }^{56} \mathrm{Fe}$, ${ }^{31} \mathrm{P},{ }^{34} \mathrm{~S},{ }^{55} \mathrm{Mn}$ were quantified with ${ }^{45} \mathrm{Sc},{ }^{89} \mathrm{Y},{ }^{115} \mathrm{In}$ as internal standards. Sulfur was used as an internal mass index as reported previously.

SEC-ICP-MS. The fractionation metal analysis of the cell lysates was performed by SEC-ICP-MS using an Agilent 1100 HPLC coupled to the ICP-MS nebulizer. The HPLC system consisted of a thermostat auto sampler kept at $4^{\circ} \mathrm{C}$, a binary pump, and a column oven kept at $25^{\circ} \mathrm{C}$, and all connections were made by $0.17 \mathrm{~mm}$ ID polyetheretherketone tubing. Different volumes of the cell lysates were injected into the HPLC based on their protein concentration. The column was a TSK3000SW from Tosoh Bioscience, with ammonium acetate $\mathrm{pH}$ 7.4 at a flow rate of $0.5 \mathrm{~mL} / \mathrm{min}$. A UV-Vis diode arrange detector was connected before the ICP-MS to evaluate the protein concentration of each sample. The ICP-MS was operated in time-resolved analysis for 32 minutes with ${ }^{66} \mathrm{Zn},{ }^{63} \mathrm{Cu},{ }^{59} \mathrm{Co},{ }^{56} \mathrm{Fe}$, and ${ }^{55} \mathrm{Mn}$ integrated at $0.2 \mathrm{sec}-$ onds per isotope. Standards were prepared from a metalloprotein mixture and injected in growing concentrations to quantify the element of interest by the external calibration method. For this, the areas of the peaks of interest were integrated after exporting the raw data into Origin software (OriginLab). The 20-minute peak was associated with MTs; the signals after 22.5 minutes were considered low molecular mass or labile fractions according to the column calibration and our previous studies (13).

Gene silencing. Genes were silenced using TransIT TKOtransfection (Mirus Bio LLC) reagent per the manufacturer's instructions. Briefly, cells were treated with siRNA-containing transfection complexes for 24 hours prior to 2-DG treatment; after that, the complexes were removed and the cells were infected and treated with 2-DG. The degree of silencing was assessed by gene expression 24 hours after infection and 2-DG treatment. The siRNA concentration was $100 \mathrm{nM}$ for all the complexes. The following siRNAs were used: ON-TARGETplus Mouse Slc39a1 siRNA (Horizon, L-055820-01-0005), ON-TARGETplus Mouse Slc39a2 siRNA (Horizon, L-041302-01-0005), and ON-TARGETplus Mouse Slc40a1 siRNA (Horizon, L-041126-01-0005).

Gene expression. For BMDMs, RNA was isolated from $1 \times 10^{6}$ BMDMs. Real-time gene expression analysis was performed using TaqMan primer/probe sets (Thermo Fisher Scientific). Expression of target genes was compared with $\beta$-2-microglobulin (B2m TaqMan Gene Expression Assays, Thermo Fisher Scientific, Mm00437762_ $\mathrm{m} 1)$ as an internal control and normalized to uninfected BMDMs. SLC39A1 TaqMan Gene Expression Assays (Thermo Fisher Scientific, Mm01605921_g1), and SLC39A2 TaqMan Gene Expression Assays (Thermo Fisher Scientific, Mm01314597_g1).

For $H$. capsulatum yeasts, qRT-PCR primer pairs used in this study were designed to yield an approximate 200 bp product. Pelleted $H$. capsulatum yeasts were resuspended in $1 \mathrm{~mL}$ of TRIzol reagent, flash-frozen in liquid nitrogen, and stored at $-80^{\circ} \mathrm{C}$ until RNA extraction. Acid-washed glass beads $(0.5 \mathrm{~mm})$ and thawed TRIzol $/ H$. capsulatum pellets were added to $2 \mathrm{~mL}$ screw-cap tubes and placed on ice. RNA was liberated by mechanical disruption using a Bead-Beater Mini (BioSpec) until approximately $80 \%$ of yeasts were lysed as determined by microscopy. A 1:10 volume of chloroform was then added to each tube, vortexed, and centrifuged at $12,000 \mathrm{~g}$ for 20 minutes at $4^{\circ} \mathrm{C}$. The aqueous fraction was then subjected to RNA clean-up and DNase treatment using the PureLink RNA Mini Kit following the manufacturer's protocol. A total of $2 \mu \mathrm{g}$ of total RNA was then reverse transcribed with the Maxima First-Strand cDNA Synthesis Kit. The cDNA was then diluted $1: 20$ and stored at $-20^{\circ} \mathrm{C}$. qRT-PCR was performed on triplicate samples using the Maxima SYBR Green/ROX qRT-PCR Master Mix on an Applied Biosystems Abi 7500-Fast Real-Time PCR System followed by melt-curve analysis. Relative gene expression was determined using the $\Delta \Delta_{\mathrm{Ct}}$ method using the constitutively expressed GAPDH housekeeping gene, and all data were normalized to H. capsulatum-only as reference (dotted line). Oligonucleotides were synthesized by Integrated DNA Technologies (Table 1).

RNA-Seq. RNA-Seq was conducted according to Rapp et al. and Walsh et al. $(53,54)$. Briefly, 500,000 BMDMs were plated in 48-well plates in RPMI plus $10 \%$ FBS. Cells were exposed to $5 \mathrm{mM}$ 2-DG or medium 1 hour before infection with $2.5 \times 10^{6} \mathrm{H}$. capsulatum yeast cells. After 3 hours, RNA was isolated. Directional RNASeq was performed by the Genomics, Epigenomics and Sequencing Core at the University of Cincinnati. The RNA concentration was measured by Nanodrop (Thermo Fisher Scientific) and its integrity analyzed by Bioanalyzer (Agilent). The NEBNext Poly(A) mRNA Magnetic Isolation Module (New England Biolabs) was used for polyA RNA purification with a total of $1 \mu \mathrm{g}$ of good-quality total RNA as input. PrepX PolyA script was employed for automated polyA RNA isolation. For the RNA-Seq library preparation, NEBNext Ultra Directional RNA Library Prep kit was used. dUTP was incorporated in second-strand synthesis to maintain strand specificity. The isolated polyA RNA or rRNA/globin-depleted RNA was fragmented with $\mathrm{Mg}^{2+}$ and heat into approximately 200 bp segments. The RNA was reverse-transcribed to cDNA followed by second-strand cDNA synthesis labeled with dUTP. The cDNA was end-repaired and dA tailed and ligated to an adapter with a stem-loop structure. The dUTP-labeled second-strand cDNA was eliminated by USER enzyme to maintain strand specificity. After indexing via PCR (11 cycles) for 


\section{Table 1. Oligonucleotides synthesized by Integrated DNA Technologies}

\begin{tabular}{|c|c|c|}
\hline Gene & Forward Primer $\left(5^{\prime}-3^{\prime}\right)$ & Reverse Primer $\left(5^{\prime}-3^{\prime}\right)$ \\
\hline GAPDH & TATTGGGCGTATTGTCTTC & GGTCTCTCTCTTGGTAGAA \\
\hline Zrt1 & TCACСТTCСАССАAАССTTCС & САTTCGTCATCСCTACCATCAG \\
\hline Zrt2 & TCСTCGAATTCGGCATCATT & ATGGAAATTGGAGTAGAGAGGC \\
\hline Zrt3 & GTGTCAGTGGCATTGCTTG & GCTAGAAGAGAGTCCAGCTTTA \\
\hline Zap1 & ССТGACACATAGTAACCAACAAAGG & CGCCAGTATCAATTCGCATGT \\
\hline
\end{tabular}

enrichment, the amplified libraries together with library preparation negative control were purified by AMPure XP beads for QC analysis. To check the yield and quality of the purified library, $1 \mu \mathrm{L}$ of the library was analyzed by Bioanalyzer using DNA high-sensitivity chip. To quantify the library concentration for the clustering, the library was diluted 1:10 in buffer (10 mM Tris- $\mathrm{HCl}, \mathrm{pH} 8.0$ with $0.05 \%$ Tween 20), and qPCR was measured by NEBNext Library Quant Kit using QuantStudio 5 Real-Time PCR Systems (Thermo Fisher Scientific).

RNA-Seq analysis. Analysis was conducted according to Chowdhury (55). Briefly, sequence reads were aligned to the reference genome using the TopHat2 aligner. Reads aligned to each known transcript were enumerated using Bioconductor packages (https:// www.bioconductor.org) for next-generation sequencing data analysis. Differential expression between different sample types was performed using the negative binomial statistical model of read counts as implemented in the edgeR Bioconductor package. Significance of differential expression was set to fold change 2 or higher and adjusted $P$ value less than 0.05. $P$ values were adjusted for multiple hypotheses testing using the Benjamini-Hochberg procedure. Each group is based on 3 biological replicates.

Data and code availability. The RNA-Seq data generated during this study are available in NCBI's Sequence Read Archive (SRA) database under accession number PRJNA668376.

Statistics. Data are represented as mean \pm SEM. Statistical analysis was performed with GraphPad Prism version 9. The $t$ test was used only to analyze differences between 2 groups. One-way ANOVA was used to test the differences between 2 or more groups with a single variable of interest. Two-way ANOVA was used to examine differences between 2 or more groups when there was more than one variable of interest. $P$ values of less than 0.05 were considered significant.

Study approval. The animal studies described herein were approved by the University of Cincinnati IACUC. For ATG conditional knockouts, we used an NIAID animal protocol that was approved by the ARC. All animal experiments were performed in accordance with the Animal Welfare Act guidelines of the NIH. The studies with human cells were conducted in accordance with the principles expressed in the Declaration of Helsinki. Deidentified whole blood was purchased from the Hoxworth Blood Center and used to prepare human macrophages. Deidentified human alveolar macrophages were obtained from patients undergoing bronchoscopy at the University of Lübeck. All procedures were performed according to German national guidelines and approved by the ethical committee of the University of Lübeck (03/153). Written informed consent was obtained.

\section{Author contributions}

DCPR wrote the manuscript, designed and performed experiments, and interpreted the data. WRB did the in vivo experiments. HME performed ECAR and OCR assays and analysis. JALF and KC performed chromatographic separations. LTB did the yeast metal transporter experiments. BLC did assays with human macrophages. WE and PRW provided BMDMs from ATG5-KO mice. $\mathrm{RL}$ did the ROS experiments and assisted with writing the manuscript. JR supervised the studies with human macrophages. GSD supervised the work.

\section{Acknowledgments}

This work was supported by NIH grants AI 106269 and AI 133797 (to GSD), International Research Training grant 1911 funded by the German Research Foundation (DFG) within project cores B8.1 and 8.2 (to JR). This work was supported by the Intramural Research Program of the National Institute of Allergy and Infectious Diseases, NIH (grants AI001123 and AIO01124 to PRW).

Address correspondence to: George S Deepe Jr., Division of Infectious Diseases, Department of Medicine, 231 Albert Sabin Way, University of Cincinnati College of Medicine, Cincinnati, Ohio 45267-0560, USA. Email: george.deepe@uc.edu.
1. Traven A, Naderer T. Central metabolic interactions of immune cells and microbes: prospects for defeating infections. EMBO Rep. 2019;20(7):e47995.

2. Thi EP, et al. Sleeping with the enemy: how intracellular pathogens cope with a macrophage lifestyle. PLoS Pathog. 2012;8(3):e1002551.

3. Deepe GS. Outbreaks of histoplasmosis: the spores set sail. PLoS Pathog. 2018;14(9):e1007213.

4. Garfoot AL, et al. Histoplasma capsulatum depends on de novo vitamin biosynthesis for intraphagosomal proliferation. Infect Immun. 2014;82(1):393-404.

5. Hilty J, et al. Histoplasma capsulatum utilizes siderophores for intracellular iron acquisition in macrophages. Med Mycol. 2011;49(6):633-642. 6. Shen $\mathrm{Q}$, et al. Macrophage activation by IFN- $\gamma$ triggers restriction of phagosomal copper from intracellular pathogens. PLoS Pathog. 2018;14(11):e1007444.

7. Shen Q, et al. Metabolism of gluconeogenic substrates by an intracellular fungal pathogen circumvents nutritional limitations within macrophages. mBio. 2020;11(2):e02712-19.

8. Billig S, et al. Lactate oxidation facilitates growth of Mycobacterium tuberculosis in human macrophages. Sci Rep. 2017;7(1):6484.

9. Czyz DM, et al. Brucella abortus induces a Warburg shift in host metabolism that is linked to enhanced intracellular survival of the pathogen. J Bacteriol. 2017;199(15):14.

10. Escoll P, et al. Legionella pneumophila modulates mitochondrial dynamics to trigger metabolic repurposing of infected macrophages. Cell Host Microbe. 2017;22(3):302-316.
11. Núñez G, et al. Innate nutritional immunity. Immunol. 2018;201(1):11-18.

12. Forbes JR, Gros P. Divalent-metal transport by NRAMP proteins at the interface of host-pathogen interactions. Trends Microbiol. 2001;9(8):397-403.

13. Subramanian Vignesh K, et al. Granulocyte macrophage-colony stimulating factor induced $\mathrm{Zn}$ sequestration enhances macrophage superoxide and limits intracellular pathogen survival. Immunity. 2013;39(4):697-710.

14. Xi H, et al. The wonders of 2-deoxy-D-glucose. IUBMB Life. 2014;66(2):110-121.

15. Datema R, Schwarz RT. Interference with glycosylation of glycoproteins. Inhibition of formation of lipid-linked oligosaccharides in vivo. Biochem J. 1979;184(1):113-123.

16. Xi H, et al. 2-Deoxy-D-glucose activates autoph- 
agy via endoplasmic reticulum stress rather than ATP depletion. Cancer Chemother Pharmacol. 2011;67(4):899-910.

17. Friedrich D, et al. The HIF-1 $\alpha /$ LC3-II axis impacts fungal immunity in human macrophages. Infect Immun. 2019;87(7):e00125-19.

18. Makis W, et al. Appearance of CNS histoplasmosis on ${ }^{1} 8$ F-FDG PET/CT with MRI correlation. BJR Case Rep. 2016;2(3):20150443.

19. Mittal B, et al. 18F-FDG PET/CT in initial staging and response assessment in patients with histoplasmosis. J Nucl Med. 2019;60(suppl 1):225.

20. Satoh T, et al. The Jmjd3-Irf4 axis regulates M2 macrophage polarization and host responses against helminth infection. Nat Immunol. 2010;11(10):936-944.

21. Eissenberg LG. Histoplasma capsulatum modulates the acidification of phagolysosomes. J Exp Med.1993;177(6):1605-1611.

22. Newman SL, et al. Human macrophages do not require phagosome acidification to mediate fungistatic/fungicidal activity against Histoplasma capsulatum. JImmunol. 2006;176(3):1806-1813.

23. Price JV, et al. Legionella pneumophila is directly sensitive to 2-deoxyglucose-phosphate via its UhpC transporter but is indifferent to shifts in host cell glycolytic metabolism. J Bacteriol. 2018;200(16):e00176-18.

24. Aft RL, et al. Evaluation of 2-deoxy-D-glucose as a chemotherapeutic agent: mechanism of cell death. Br J Cancer. 2002;87(7):805-812.

25. Michelakis ED, et al. Dichloroacetate (DCA) as a potential metabolic-targeting therapy for cancer. Br J Cancer. 2008;99(7):989-994.

26. Weinberg F, et al. Mitochondrial metabolism and ROS generation are essential for Krasmediated tumorigenicity. Proc Natl Acad Sci US A. 2010;107(19):8788-8793.

27. Kimmey JM, Stallings CL. Bacterial pathogens versus autophagy: implications for therapeutic interventions. Trends $\mathrm{Mol} \mathrm{Med}$. 2016;22(12):1060-1076

28. Huang JH, et al. NLRX1 facilitates histoplasma capsulatum-induced LC3-associated phagocytosis for cytokine production in macrophages. Front Immunol. 2018;9:2761.

29. Matsuda F, et al. Autophagy induced by 2-deoxy$\mathrm{D}$-glucose suppresses intracellular multiplication of Legionella pneumophila in A/J mouse macro- phages. Autophagy. 2009;5(4):484-493.

30. Galluzzi L, Green DR. Autophagy-independent functions of the autophagy machinery. Cell. 2019;177(7):1682-1699.

31. Kropski JA, Blackwell TS. Endoplasmic reticulum stress in the pathogenesis of fibrotic disease. J Clin Invest. 2018;128(1):64-73.

32. Wang $\mathrm{M}$, et al. Role of the unfolded protein response regulator GRP78/BiP in development, cancer, and neurological disorders. Antioxid Redox Signal. 2009;11(9):2307-2316.

33. Kurtoglu M, et al. Differential toxic mechanisms of 2-deoxy-D-glucose versus 2-fluorodeoxyD-glucose in hypoxic and normoxic tumor cells. Antioxid Redox Signal. 2007;9(9):1383-1390.

34. Hwang LH, et al. Histoplasma requires SID1, a member of an iron-regulated siderophore gene cluster, for host colonization. PLoS Pathog. 2008;4(4):e1000044

35. Newman SL, et al. Chloroquine induces human macrophage killing of Histoplasma capsulatum by limiting the availability of intracellular iron and is therapeutic in a murine model of histoplasmosis. J Clin Invest. 1994;93(4):1422-1429.

36. Newman SL, et al. Inhibition of growth of Histoplasma capsulatum yeast cells in human macrophages by the iron chelator VUF 8514 and comparison of VUF 8514 with deferoxamine. Antimicrob Agents Chemother. 1995;39(8):1824-1829.

37. Maret W. Redox biochemistry of mammalian metallothioneins. J Biol Inorg Chem. 2011;16(7):1079-1086.

38. Hu J. Towards unzipping the ZIP metal transporters: structure, evolution, and implications on drug discovery against cancer [published online December 9, 2020]. FEBS J. https://doi. org/10.1111/febs.15658.

39. Gleeson LE, et al. Cutting edge: Mycobacterium tuberculosis induces aerobic glycolysis in human alveolar macrophages that is required for control of intracellular bacillary replication. J Immunol. 2016;196(6):2444-2449.

40. Huang L, et al. Growth of Mycobacterium tuberculosis in vivo segregates with host macrophage metabolism and ontogeny. JExp Med. 2018;215(4):1135-1152.

41. Braverman J, et al. HIF- $1 \alpha$ is an essential mediato of IFN- $\gamma$-dependent immunity to Mycobacterium tuberculosis. JImmunol. 2016;197(4):1287-1297.
42. Wang A, et al. Opposing effects of fasting metabolism on tissue tolerance in bacterial and viral inflammation. Cell. 2016;166(6):1512-1525.

43. Wang A, et al. Glucose metabolism mediates disease tolerance in cerebral malaria. Proc Natl Acad Sci U S A. 2018;115(43):11042-11047.

44. Passalacqua KD, et al. Glycolysis is an intrinsic factor for optimal replication of a norovirus. mBio. 2019;10(2):e02175-18.

45. Grootjans J, et al. The unfolded protein response in immunity and inflammation. Nat Rev Immunol. 2016;16(8):469-484

46. Rashid HO, et al. ER stress: autophagy induction, inhibition and selection. Autophagy. 2015;11(11):1956-1977.

47. Winters MS, et al. Metallomic analysis of macrophages infected with Histoplasma capsulatum reveals a fundamental role for zinc in host defenses. J Infect Dis. 2010;202(7):1136-1145.

48. Garin J et al. The phagosome proteome: insight into phagosome functions. J Cell Biol. 2001;152(1):16-180.

49. Maret W. Inhibitory zinc sites in enzymes. Biometals. 2013;26(2):197-204.

50. Subramanian Vignesh K, et al. IL-4 induces metallothionein 3- and SLC30A4-dependent increase in intracellular $\mathrm{Zn}^{2+}$ that promotes pathogen persistence in macrophages. Cell Reports. 2016;16(12):3232-3246.

51. Visse R, Nagase H. Matrix metalloproteinases and tissue inhibitors of metalloproteinases: structure, function, and biochemistry. Circ Res. 2003;92(8):827-839.

52. Scott AJ, Woods JP. Monitoring internalization of Histoplasma capsulatum by mammalian cell lines using a fluorometric microplate assay. Med Mycol. 2000;38(1):15-22.

53. Rapp SJ, et al. Varying negative pressure wound therapy acute effects on human split-thickness autografts. J Burn Care Res. 2019;41(1):104-112.

54. Walsh KB, et al. Intracerebral hemorrhage induces inflammatory gene expression in peripheral blood: global transcriptional profiling in intracerebral hemorrhage patients. DNA Cell Biol. 2019;38(7):660-669.

55. Chowdhury D, et al. Metallothionein 3 controls the phenotype and metabolic programming of alternatively activated macrophages. Cell Rep. 2019;27(13):3873-3886. 